\title{
Torsion points on elliptic curves in Weierstrass form
}

\author{
PHILIPP HABEGGER
}

\begin{abstract}
We prove that there are only finitely many complex numbers $a$ and $b$ with $4 a^{3}+27 b^{2} \neq 0$ such that the three points $(1, *),(2, *)$, and $(3, *)$ are simultaneously torsion points on the elliptic curve defined in Weierstrass form by $y^{2}=x^{3}+a x+b$. This gives an affirmative answer to a question raised by Masser and Zannier. We thus confirm a special case in two dimensions of the relative Manin-Mumford Conjecture formulated by Pink and Masser-Zannier.
\end{abstract}

Mathematics Subject Classification (2010): 14H52 (primary); 14G40, 11G05, 11U09 (secondary).

\section{Main Result}

In pursuit of unlikely intersections, Masser and Zannier [10,11] proved that there are only finitely many complex $\lambda \neq 0,1$ such that

$$
(2, \sqrt{2(2-\lambda)}) \text { and }(3, \sqrt{6(3-\lambda)})
$$

are torsion points on the elliptic curve given in Legendre form by $y^{2}=x(x-1)(x-$ $\lambda)$.

This result provides evidence for far-reaching conjectures stated by its authors $[9,11]$ and by Pink [14]. Both conjectures govern the distribution of torsion points on a subvariety of a family of semi-Abelian varieties and may be regarded as a relative version of the Manin-Mumford Conjecture. They deal with unlikely or anomalous intersections emphasized in the earlier work of Zilber [22] for constant semi-Abelian varieties. In Masser and Zannier's result the subvariety is an algebraic curve inside the fibered square of the Legendre family of elliptic curves. By a recent example of Bertrand [3], these conjectures require modification when dealing with families whose fibers are not complete. However, in the current paper we will consider only families of Abelian varieties.

One natural family is the Weierstrass family. The Weierstrass equation $y^{2}=$ $x^{3}+a x+b$ defines an elliptic curve when $a$ and $b$ are complex parameters that 
satisfy the inequality $4 a^{3}+27 b^{2} \neq 0$ which rules out singularities. We thus obtain a family of elliptic curves parametrized by $a$ and $b$. Masser and Zannier [11] asked if a similar finiteness statement as above holds in this context. Because there are two parameters, the conjectures suggest imposing a torsion condition on a third point to expect finiteness.

Our main result gives a positive answer to Masser and Zannier's question and provides the first evidence supporting a relative Manin-Mumford Conjecture over a base of dimension greater than one.

Theorem 1.1. There are only finitely many complex pairs $(a, b)$ with $4 a^{3}+27 b^{2} \neq$ 0 such that

$$
(1, \sqrt{1+a+b}), \quad(2, \sqrt{8+2 a+b}), \quad \text { and } \quad(3, \sqrt{27+3 a+b})
$$

are torsion points on the elliptic curve given in Weierstrass form $y^{2}=x^{3}+a x+b$.

Although the methods we present are as a whole confined to a specific example, some intermediate steps hold in greater generality. It is therefore convenient to work in a more general language. When not stated otherwise, a variety is defined over C. We also identify a variety with the set of its complex points. If a variety $X$ is defined over a field $K$ it is sometimes still useful to write $X(K)$ for the $K$-rational points on $X$. surface

We proceed by reformulating our main result. Let $S$ be the affine algebraic

$$
\left\{(a, b) \in \mathbf{A}^{2} ; 4 a^{3}+27 b^{2} \neq 0\right\} ;
$$

it is defined over $\overline{\mathbf{Q}}$, the algebraic closure of $\mathbf{Q}$ in $\mathbf{C}$. The Weierstrass family of elliptic curves

$$
\mathcal{E}=\left\{([x: y: z],(a, b)) \in \mathbf{P}^{2} \times S ; y^{2} z=x^{3}+a x z^{2}+b z^{3}\right\}
$$

is an Abelian scheme over the two-dimensional base $S$. Let $\mathcal{E}^{3}$ be the three-fold fibered power of $\mathcal{E}$ over $S$ and $\pi: \mathcal{E}^{3} \rightarrow S$ by the structure morphism. We obtain an Abelian scheme over $S$. A complex point of an Abelian scheme that is torsion in its respective fiber will be called a torsion point.

In this language, our result states that all torsion points on a certain, explicitly given, algebraic surface $X \subset \mathcal{E}^{3}$ are contained in finitely many fibers of $\mathcal{E}^{3} \rightarrow S$ This surface, we call it the 123-surface, is the Zariski closure of the affine subset of $\mathcal{E}^{3}$ where the first coordinate in each copy of $\mathcal{E}$ is fixed to be 1,2 , and 3 , respectively. The restriction of $\mathcal{E}^{3} \rightarrow S$ to $X$ has finite fibers, so our main result is equivalent to the statement that $X$ contains only finitely many torsion points.

The general conjecture stated by Masser and Zannier [11] in the case of families of Abelian varieties expects the torsion points on our surface to lie on finitely many proper subgroup schemes of $\mathcal{E}^{3}$. If true, it could at best imply that torsion points do not lie Zariski dense on $X$. Our Theorem 1.1 however, is unconditional. 
Moreover, our finiteness statement is stronger than the conjecture's conclusion. This feature is due to the specific nature of our surface.

Let us consider for the moment a variation of the 123-surface. We claim that there are infinitely many complex $(a, b) \in S$ such that

$$
(0, \sqrt{b}), \quad(1, \sqrt{1+a+b}), \quad \text { and } \quad(-1, \sqrt{-1-a+b})
$$

are torsion points on the elliptic curve $y^{2}=x^{3}+a x+b$. Indeed, we find them on $b=0$. The first point is automatically torsion of order 2 . We observe that $y^{2}=x^{3}+a x$ yields an elliptic curve with complex multiplication and $j$-invariant 1728. It follows from basic facts on elliptic curves that there are infinitely many $a \in \mathbf{C} \backslash\{0\}$ such that $(1, \sqrt{1+a})$ is torsion on $y^{2}=x^{3}+a x$; we shall prove a related statement in Lemma 3.9. We fix such an $a$. Then $(-1, \sqrt{-1-a})$ is the image of $(1, \sqrt{1+a})$ under an automorphism of order 4 of $y^{2}=x^{3}+a x$. So all three points in (1.3) are torsion. Using a specialization argument one can show that the algebraic surface in $\mathcal{E}^{3}$ induced by (1.3) is not in a proper subgroup scheme of $\mathcal{E}^{3}$. Conjecturally, it does not contain a Zariski dense set of torsion points.

Let us briefly recap the proof of Theorem 1.1. It splits up into two parts. In the first half, laid out in Section 2, we work in the Legendre family of elliptic curves

$$
\mathcal{E}_{L}=\left\{([x: y: z], \lambda) \in \mathbf{P}^{2} \times Y(2) ; y^{2} z=x(x-z)(x-\lambda z)\right\}
$$

where $Y(2)=\mathbf{P}^{1} \backslash\{0,1, \infty\}$. The three-fold fibered power of $\mathcal{E}_{L} \rightarrow Y(2)$ is denoted by $\mathcal{E}_{L}^{3}$. Working in the Legendre family has the advantage that the base is one dimensional.

Any elliptic curve over $\mathbf{C}$ is isomorphic to an elliptic curve in Legendre form. Using a base change argument we can construct a new algebraic surface in $\mathcal{E}_{L}^{3}$ using the 123-surface. The study of torsion points on the 123 -surface will be carried out by studying torsion points on this new surface.

The first part of the proof makes no use of the special form of the 123-surface. So all partial results will be formulated for an arbitrary irreducible algebraic surface $X_{L}$ in $\mathcal{E}_{L}^{3}$.

On any elliptic curve, or more generally, on any Abelian scheme we use $[N]$ to denote the multiplication by $N \in \mathbf{Z}$ morphism. In Proposition 2.1, we prove that $X_{L}$ contains only finitely many torsion points outside the so-called torsion anomalous locus of $X_{L}$. Informally, this is the union of all positive dimension subvarieties of $X_{L}$ on which an excessive number of independent integral relations

$$
\begin{aligned}
{[\alpha]\left(P_{1}\right)+[\beta]\left(P_{2}\right)+[\gamma]\left(P_{3}\right)=0 } & \text { where } \quad\left(P_{1}, P_{2}, P_{3}, \lambda\right) \in X_{L} \\
& \text { and } \alpha, \beta, \gamma \in \mathbf{Z}
\end{aligned}
$$

hold identically. A precise definition is provided in Section 2.

To prove Proposition 2.1 we follow the basic strategy originally proposed by Zannier. It involves estimating from above and below the number of rational points 
on certain sufficiently tame sets. This strategy already appeared in the proof of Masser and Zannier's result mentioned further up. It was also used in a new proof of the Manin-Mumford Conjecture by Pila and Zannier [13].

An elliptic logarithm of a torsion point on an elliptic curve has rational coefficients with respect to a chosen period lattice basis. The conjugate of any torsion point again leads to a rational point. This observation together with estimates for the Galois orbit of a torsion point yields the required lower bounds for rational points. Masser and Zannier required an upper bound, proved by Pila, for the number of rational points with fixed denominator on compact subanalytic surfaces.

Additional difficulties arise in our situation since $X_{L}$ is an algebraic surface as opposed to the algebraic curve connected with (1.1). For example, a crucial height inequality used by Masser and Zannier which depends on work of Silverman has only recently been extended to higher dimension [6] by the author.

Algebraic independence statements for certain transcendental functions related to elliptic logarithms played an important role in Masser and Zannier's result regarding (1.1) and even more so in their generalization to curves [9]. These cannot be applied directly to the higher dimensional case; and neither can Bertrand's more general results [2]. We overcome this difficulty using two tools. First, we use a bound of David [5] on the number of torsion points defined over a number field on an elliptic curve. The quality of his bound is indispensable in our method. It enables us to choose a "wandering curve" in $X_{L}$ containing sufficiently many conjugates of a given torsion point. We can then apply results from the one dimensional case to this curve. Second, we replace Pila's counting result by the powerful theorem of Pila and Wilkie [12] formulated in the versatile language of o-minimal structures. This additional generality is required to treat the real 4 dimensional sets which arise naturally in our problem. The Pila-Wilkie Theorem is uniform over definable families, a feature which is needed to control the wandering curve constructed above.

A brief recollection of the theory of o-minimal structures is presented in Subsection 2.1. Using David's result we will find an abundance of rational points coming from elliptic logarithms on one fiber of a definable family. Enough actually, to successfully compete with the upper bound coming from the Pila-Wilkie Theorem.

In the second half of the proof, detailed in Section 3, we return to the Weierstrass family, the natural setting of our main result. The obstruction to obtaining finiteness in the first half was the torsion anomalous locus of $X_{L}$. There is also an analogous locus for algebraic surfaces in $\mathcal{E}^{3}$. The goal of the second half is to get hold of this locus for the 123-surface. In fact, Proposition 3.2 tells us that it is empty. We briefly indicate the general idea of the argument.

Typically, an anomalous subvariety is an irreducible algebraic curve $C \subset X$ on which two independent relations as in (1.4) hold. We can specialize to any point in the image of $C$ under $\mathcal{E}^{3} \rightarrow S$. This yields three points on an elliptic curve over $\mathbf{C}$ which are connected by two independent relations. In this situation it seems difficult to directly extract information from the fact that the first affine coordinates of these three points are 1,2, and 3. Roughly speaking, we will specialize to a point on the boundary of a compactification of $S$. Let us consider the morphism 
$C \rightarrow \pi(C)$ coming from the restriction to $C$ of $\pi: \mathcal{E}^{3} \rightarrow S$. Passing to the generic fiber yields a point on the cube of an elliptic curve defined over the function field of $\pi(C)$. Let us assume, for now, that this elliptic curve has a place of split multiplicative reduction. We use the Tate uniformization which relates the group structure of an elliptic curve and the multiplicative group of a field. This will allow us to translate the excessive number of integral relations into a completely explicit multiplicative relation involving algebraic numbers derived from 1,2 , and 3 . It is then a simple matter to show that this multiplicative relation is untenable. From this we will deduce that the generic fiber of $C \rightarrow \pi(C)$ must have good reduction everywhere. Therefore, all fibers share a common $j$-invariant. From this severe restriction it will not be difficult to derive a contradiction using the particular nature of the 123-surface.

We make heavy use of the special nature of our surface in the second half. What happens if one replaces 1,2, 3 by another triple of algebraic numbers? We have seen that finiteness need not hold even if the triple consists of pairwise distinct integers. In an unpublished manuscript the author described a necessary condition on the triple to ensure a finiteness statement as in Theorem 1.1. For example, the first three primes 2,3,5 also yield a finiteness result as in our main result.

The author is very grateful to David Masser and Umberto Zannier for the numerous conversations, especially productive in Pisa, July 2010. He also thanks the latter for the invitation to Pisa and the Scuola Normale Superiore for its hospitality and financial support. The author was also supported by SNSF project number 124737.

\section{Torsion points outside the torsion anomalous locus}

We will work with an irreducible closed algebraic surface $X$ in $\mathcal{E}_{L}^{3}$. The 123-surface will not appear in the current section. So no ambiguity can occur if we avoid the more cumbersome notation $X_{L}$ from the introduction and use $\pi$ to denote the projection $\mathcal{E}_{L} \rightarrow Y(2)$. We do keep the subscript in $\mathcal{E}_{L}$ to emphasize that we are in the Legendre family.

For $\lambda \in Y(2)$ the fiber $\left(\mathcal{E}_{L}\right)_{\lambda}=\pi^{-1}(\lambda)$ is taken as an elliptic curve given in Legendre form. We identify the three-fold fibered power $\mathcal{E}_{L}^{3}$ of $\mathcal{E}_{L} \rightarrow Y(2)$ with

$$
\mathcal{E}_{L}^{3}=\left\{\left(P_{1}, P_{2}, P_{3}, \lambda\right) \in\left(\mathbf{P}^{2}\right)^{3} \times Y(2) ; P_{1}, P_{2}, P_{3} \in\left(\mathcal{E}_{L}\right)_{\lambda}\right\} .
$$

By abuse of notation we also use $\pi$ for the projection $\mathcal{E}_{L}^{3} \rightarrow Y(2)$ and write $\left(\mathcal{E}_{L}^{3}\right)_{\lambda}=$ $\pi^{-1}(\lambda) \subset \mathcal{E}_{L}^{3}$. Recall that $\left(P_{1}, P_{2}, P_{3}, \lambda\right) \in \mathcal{E}_{L}^{3}$ is called torsion if $P_{1}, P_{2}$, and $P_{3}$ are torsion points of $\left(\mathcal{E}_{L}\right)_{\lambda}$.

Any $\chi=(\alpha, \beta, \gamma) \in \mathbf{Z}^{3}$ determines a Zariski closed set $G_{\chi} \subset \mathcal{E}_{L}^{3}$ through the integral relation

$$
[\alpha]\left(P_{1}\right)+[\beta]\left(P_{2}\right)+[\gamma]\left(P_{3}\right)=0 .
$$


An irreducible closed subvariety $A$ of $X$ is called a torsion anomalous subvariety of $X$

(i) if $\operatorname{dim} A=1$ and two independent integral relations hold on $A$,

(ii) or if $\operatorname{dim} A=2$ and one non-trivial integral relation holds on $A$,

(iii) or if $\operatorname{dim} A \geq 1$ and $A$ is an irreducible component of an algebraic subgroup of $\left(\mathcal{E}_{L}^{3}\right)_{\lambda}$ for some $\lambda \in Y(2)$ such that $\left(\mathcal{E}_{L}\right)_{\lambda}$ has complex multiplication.

The torsion anomalous locus of $X$ is $\bigcup_{A} A$, here $A$ runs over all torsion anomalous subvarieties of $X$. We write $X^{\text {ta }}$ for the complement of the torsion anomalous locus in $X$.

An irreducible closed subvariety $A \subset \mathcal{E}_{L}^{3}$ which dominates $Y(2)$ is called a component of a flat subgroup scheme of $\mathcal{E}_{L}$

(i) if $\operatorname{dim} A=1$ and three independent integral relations hold on $A$,

(ii) or if $\operatorname{dim} A=2$ and two independent integral relation hold on $A$,

(iii) or if $\operatorname{dim} A=3$ and one independent integral relation holds on $A$,

(iv) or if $A=\mathcal{E}_{L}^{3}$.

We write $X^{\star}$ for $X \backslash \bigcup_{A} A$, here $A$ runs over all components of flat subgroup schemes of $\mathcal{E}_{L}$ contained completely in $X$. We have $X^{\text {ta }} \subset X^{\star}$.

The definition of $X^{\star}$ coincides with the complex points of the corresponding definition given in [6]. Indeed, see Lemma 2.5 in this reference.

The purpose of this section is to prove that there are only finitely many points outside the torsion anomalous locus of $X$.

Proposition 2.1. Let $X \subset \mathcal{E}_{L}^{3}$ be an irreducible closed algebraic surface defined over $\overline{\mathbf{Q}}$ which dominates $Y(2)$.

(i) There are at most finitely many torsion points in $X^{\mathrm{ta}}$.

(ii) The set

$$
\left\{\pi(P) ; P \in X^{\star} \text { is torsion and }\left(\mathcal{E}_{L}\right)_{\pi(P)} \text { has complex multiplication }\right\}
$$

is finite.

It is conceivable that the union in the definition of $X \backslash X^{\text {ta }}$ or $X \backslash X^{\star}$ is over infinitely many $A$. So we have no reason to expect that $X^{\text {ta }}$ or $X^{\star}$ is Zariski open. However, $X^{\star}$ is known to be Zariski open by [6, Theorem 1.3(i)]. In a later section we will address the problem of describing $X^{\text {ta }}$ for an algebraic surface derived from the 123-surface. In this particular situation, $X^{\text {ta }}$ will be Zariski open.

The author believes that $X^{\text {ta }}$ is Zariski open for all surfaces. More precisely, he expects $X$ to contain only finitely many torsion anomalous subvarieties that are not strictly contained in another torsion anomalous subvariety of $X$.

Let us assume for the moment that this finiteness statement holds for $X$. Let us also assume that no non-trivial integral relation holds identically on $X$ and that $X$ dominates $Y(2)$. In this case we sketch how Proposition 2.1 implies a uniform 
Manin-Mumford-type statement in a family of Abelian varieties. Indeed, we may regard $X$ as a family of curves $\left\{X_{\lambda}=X \cap \pi^{-1}(\lambda)\right\}$ parametrized by $\lambda \in Y(2)$. Upto finitely many exceptions, controlled by the proposition, any torsion point on a member of this family lies on one of finitely many anomalous subvariety as in cases (i) and (iii) of the definition. So any torsion point on $X$ satisfies two independent relations coming from a fixed finite set. If we are in case (i) then these relations are integral; in case (iii) they have coefficients in the endomorphism ring of an elliptic curve with complex multiplication. It is not difficult to deduce that $X_{\lambda}$ contains a positive dimensional irreducible component of an algebraic subgroup for at most finitely many $\lambda$. For all other $\lambda$ two independent relations as above intersect $X_{\lambda}$ in a finite set whose cardinality can be bounded from above independently of $\lambda$ using Bézout's Theorem. We conclude that after omitting finitely many $\lambda$ there is a uniform upper bound for the number of torsion points on $X_{\lambda}$.

In the remainder of this section we will assume that $X$ is as in the proposition. So it dominates $Y(2)$ and we may fix a number field $F \subset \overline{\mathbf{Q}}$ over which it is defined.

We will work with real parameters $B \geq 1$ and $\delta \in(0,1]$. Here $\delta$ may depend on $B$ and $B$ may depend on the surface $X$ and on $F$. If not stated otherwise, the symbols $c_{1}, c_{2}, \ldots$ will denote positive constants which may depend $X, F, \delta$, and $B$. During the proof $B$ and $\delta$ will be chosen properly.

\section{1. o-minimal structures}

We provide the definition of an o-minimal structure. For an in-depth treatment of this subject we refer to van den Dries's book [21].

Let $\mathbf{N}=\{1,2,3, \ldots\}$. An o-minimal structure is a sequence $\mathfrak{S}=\left(S_{1}, S_{2}, \ldots\right)$ such that if $n, m \in \mathbf{N}$ then $S_{n}$ is a collection of subsets of $\mathbf{R}^{n}$ with the following properties.

(i) The intersection of two sets in $S_{n}$ is in $S_{n}$ and the complement of a set in $S_{n}$ is in $S_{n}$.

(ii) Any real semi-algebraic subset of $\mathbf{R}^{n}$ is in $S_{n}$.

(iii) The Cartesian product of a set in $S_{n}$ with a set in $S_{m}$ is in $S_{n+m}$.

(iv) The image of a set in $S_{n+m}$ under the projection $\mathbf{R}^{n} \times \mathbf{R}^{m} \rightarrow \mathbf{R}^{n}$ onto the first $n$ coordinates is in $S_{n}$.

(v) A set in $S_{1}$ is a finite union of points and open, possibly unbounded, intervals.

The first four properties assert that an o-minimal structure contains enough interesting sets to work with. The fifth property restricts the possible sets in all $S_{n}$ because these project to $\mathbf{R}$ by (iv).

We call a subset of $\mathbf{R}^{n}$ definable in $\mathfrak{S}$ if it lies in $S_{n}$. If $X \subset \mathbf{R}^{n}$ then we call a function $f: X \rightarrow \mathbf{R}^{m}$ definable in $\mathfrak{S}$ if its graph, a subset of $\mathbf{R}^{n} \times \mathbf{R}^{m}$, lies in $S_{n+m}$. Domain and image of a function that is definable in $\mathfrak{S}$ are definable in $\mathfrak{S}$.

A subset $Z$ of $\mathbf{R}^{n} \times \mathbf{R}^{m}$ that is definable in $\mathfrak{S}$ is sometimes called a family definable in $\mathfrak{S}$. We do this to emphasizes that $Z$ can be seen as a collection of subsets of $\mathbf{R}^{n}$ parametrized by $\mathbf{R}^{m}$. Concretely, for $y \in \mathbf{R}^{m}$ we let $Z_{y}$ denote the projection of $Z \cap\left(\mathbf{R}^{n} \times\{y\}\right)$ to $\mathbf{R}^{n}$. Then $Z_{y}$ is definable in $\mathfrak{S}$. 
To formulate the result of Pila and Wilkie mentioned in the introduction, we shall define the exponential Weil height on the rational numbers by setting $H(p / q)=\max \{|p|, q\}$ for coprime integers $p$ and $q$ with $q \geq 1$. In higher dimension we set $H\left(\xi_{1}, \ldots, \xi_{n}\right)=\max \left\{H\left(\xi_{1}\right), \ldots, H\left(\xi_{n}\right)\right\}$ for $\left(\xi_{1}, \ldots, \xi_{n}\right) \in \mathbf{Q}^{n}$ Let $X \subset \mathbf{R}^{n}$ be any subset for the moment. The counting function associated to $X$ is

$$
N(X, T)=\#\left\{\xi \in X \cap \mathbf{Q}^{n} ; H(\xi) \leq T\right\} \text { for } T \geq 1 ;
$$

there are only finitely many points in $\mathbf{Q}^{n}$ of bounded height, so the cardinality is finite.

We define $X^{\text {alg }} \subset X$ to be the union of all connected, positive dimensional real semi-algebraic sets contained in $X$.

Theorem 2.2 (Pila-Wilkie [12]). Let $Z \subset \mathbf{R}^{n} \times \mathbf{R}^{m}$ be a family definable in an $o$-minimal structure and let $\epsilon>0$. There is a constant $c>0$ depending on $Z$ and $\epsilon$ such that if $y \in \mathbf{R}^{m}$, then

$$
N\left(Y \backslash Y^{\mathrm{alg}}, T\right) \leq c T^{\epsilon} \quad \text { for all } \quad T \geq 1
$$

where $Y=Z_{y}$.

By the Tarski-Seidenberg Theorem, the collection of all real semi-algebraic sets satisfies (iv) in the definition of an o-minimal structure. From this it is not difficult to show that the real semi-algebraic sets define an o-minimal structure. But this structure is not large enough for our needs. Luckily, a variety of larger o-minimal structures are known. For example, van den Dries [20] reinterpreted a result of Gabrielov as stating that the so-called finitely subanalytic sets form an ominimal structure $\mathbf{R}_{\mathrm{an}}$. We will not give the definition of such sets here. It suffices to remark that the restriction to $[-1,1]^{n}$ of a real valued analytic function on a neighborhood of $[-1,1]^{n}$ is definable in $\mathbf{R}_{\mathrm{an}}$. This will be enough functions for our application.

For the remainder of this section we will call sets, functions, and families $d e$ finable if they are definable in $\mathbf{R}_{\text {an }}$.

We could not find a reference for the following, possibly well-known, statement. Therefore, we provide its short proof which is valid in any o-minimal structure.

Lemma 2.3. Let $X \subset \mathbf{R}^{n}$ be a definable set and let $f: X \rightarrow \mathbf{R}^{m}$ be a definable function. There are definable sets $X_{0}, \ldots, X_{M} \subset \mathbf{R}^{n}$ with $X=X_{0} \cup X_{1} \cup \cdots \cup X_{M}$ such that $\left.f\right|_{X_{1}}, \ldots,\left.f\right|_{X_{M}}$ are injective and such that the fibers of $\left.f\right|_{X_{0}}$ contain no isolated points. Here $X_{0}=\emptyset$ and $M=0$ are possible.

Proof. We first prove the lemma when $f$ has finite fibers. Then the fibers have cardinality bounded from above uniformly by [21, Corollary 3.6, page 60]. Say $c$ is the maximal cardinality attained. We may suppose $c \geq 2$. By Definable Choice, Proposition 1.2, page $93 \mathrm{ibid}$., there is a definable function $g: f(X) \rightarrow \mathbf{R}^{n}$ with $f(g(y))=y$ for all $y \in f(X)$. The sets $g(f(X))$ and $X \backslash g(f(X))$ are definable. 
Now the definable function $\left.f\right|_{g(f(X))}$ is injective and the fibers of the definable function $\left.f\right|_{X \backslash g(f(X))}$ have cardinality at most $c-1$. The current case of the lemma follows by induction on $c$.

In the general case we observe that

$$
X_{0}=\left\{x \in X ; x \text { not isolated in } f^{-1}(f(x))\right\}
$$

is a definable set by the Cell Decomposition Theorem, $c f$. page $52 \mathrm{ibid}$. We note that $X_{0}$ contains no isolated points. The function $f$ restricted to its complement in $X$ has discrete fibers. Again by Corollary 3.6, page $60 \mathrm{ibid}$. these fibers are finite. This enables us to reduce to the situation above.

\subsection{A definable family}

In the current subsection, any reference to a topology on $X$ or $\mathcal{E}_{L}^{3}$ will refer to the Euclidean topology if not stated otherwise.

In a neighborhood of $1 / 2 \in Y(2)=\mathbf{C} \backslash\{0,1\}$ we may describe a period lattice basis of the fiber of $\mathcal{E}_{L}$ using Gauss's hypergeometric function, $c f$. [8, Chapter 9 ]. This period lattice basis can be continued analytically along any path in $Y(2)$. We fix a path from any point in $\mathcal{E}_{L}^{3}$ to the zero element of $\left(\mathcal{E}_{L}^{3}\right)_{1 / 2}$. We continue the periods along the path induced in $Y(2)$.

Any $P \in \mathcal{E}_{L}^{3}$ has a neighborhood $V_{P}$ in $\mathcal{E}_{L}^{3}$ on which we may choose holomorphic elliptic logarithms

$$
z_{P 1}, z_{P 2}, z_{P 3}: V_{P} \rightarrow \mathbf{C} .
$$

We may also fix holomorphic functions $f_{P}, g_{P}: V_{P} \rightarrow \mathbf{C}$ whose values determine a basis of the period lattice of the corresponding fiber.

The values of $f_{P}$ and $g_{P}$ are $\mathbf{R}$-linearly independent. We can express $z_{P k}$ in terms of $f_{P}$ and $g_{P}$ using real analytic functions $\xi_{P 1}, \ldots, \xi_{P 6}: V_{P} \rightarrow \mathbf{R}$, i.e.

$z_{P 1}=\xi_{P 1} f_{P}+\xi_{P 2} g_{P}, \quad z_{P 2}=\xi_{P 3} f_{P}+\xi_{P 4} g_{P}, \quad$ and $\quad z_{P 3}=\xi_{P 5} f_{P}+\xi_{P 6} g_{P}$.

We write $\theta_{P}: V_{P} \rightarrow \mathbf{R}^{6}$ for the real analytic function

$$
Q \mapsto\left(\xi_{P 1}(Q), \ldots, \xi_{P 6}(Q)\right) .
$$

It provides coordinates of an elliptic logarithm of $Q$ in terms of the period lattice basis given by $f_{P}(Q)$ and $g_{P}(Q)$.

After shrinking $V_{P}$ we may suppose that it is contained in an affine subset of $\mathcal{E}_{L}^{3}$. This has the effect that if $X^{\prime} \subset \mathcal{E}_{L}^{3}$ is Zariski closed then $X^{\prime} \cap V_{P}$ can be described as the set of common zeros of finitely many polynomials restricted to $V_{P}$.

We note that $\mathcal{E}_{L}^{3}$ is an 8-dimension real analytic manifold. After shrinking $V_{P}$ there is a real bianalytic map $\vartheta_{P}:(-2,2)^{8} \rightarrow V_{P}$ taking 0 to $P$. We define

$$
U_{P}=X \cap \vartheta_{P}\left([-1,1]^{8}\right) \subset V_{P} .
$$


Then $U_{P}$ is compact since $\vartheta_{P}\left([-1,1]^{8}\right)$ is compact. It is also a neighborhood of $P$ in $X$.

The compact set

$$
\Lambda_{\delta}=\left\{z \in \mathbf{C} ; \delta \leq|z| \leq \delta^{-1} \quad \text { and } \quad|1-z| \geq \delta\right\}
$$

is contained in $Y(2)$. The pre-image $\left.\pi\right|_{X} ^{-1}\left(\Lambda_{\delta}\right)=X \cap\left(\left(\mathbf{P}^{2}\right)^{3} \times \Lambda_{\delta}\right)$ is also compact. This set is covered by all neighborhoods $U_{P}$ with $\left.P \in \pi\right|_{X} ^{-1}\left(\Lambda_{\delta}\right)$. So there is a positive integer $c_{1}$ and $P_{1}, \ldots,\left.P_{c_{1}} \in \pi\right|_{X} ^{-1}\left(\Lambda_{\delta}\right)$ with $\left.U_{P_{1}} \cup \cdots \cup U_{P_{c_{1}}} \supset \pi\right|_{X} ^{-1}\left(\Lambda_{\delta}\right)$.

In the following, we drop the $P$ and write $U_{i}, V_{i}, \theta_{i}, \vartheta_{i}$ for $U_{P_{i}}, V_{P_{i}}, \theta_{P_{i}}, \vartheta_{P_{i}}$, respectively.

Let $|\cdot|$ denote the maximum norm on $\mathbf{R}^{n}$.

Lemma 2.4. Let $1 \leq i \leq c_{1}$. There are sets $U_{i 0}, \ldots, U_{i M_{i}}$ with $U_{i}=U_{i 0} \cup \ldots \cup$ $U_{i M_{i}}$ such that the following properties hold.

(i) The functions $\left.\theta_{i}\right|_{U_{i 1}}, \ldots,\left.\theta_{i}\right|_{U_{i M_{i}}}$ are injective and the fibers of $\left.\theta_{i}\right|_{U_{i 0}}$ contain no isolated points.

(ii) If $X^{\prime} \subset \mathcal{E}_{L}^{3}$ is Zariski closed, then $\theta_{i}\left(X^{\prime} \cap U_{i j}\right) \subset \mathbf{R}^{6}$ is definable for all $0 \leq j \leq M_{i}$.

(iii) There is $c_{2}$ with $|\xi| \leq c_{2}$ if $\xi \in \theta_{i}\left(U_{i}\right)$.

Proof. By construction, $X \cap V_{i}$ is the zero set in $V_{i}$ of functions that are polynomial on $V_{i}$. So each pre-image $\vartheta_{i}^{-1}\left(U_{i}\right)=\vartheta_{i}^{-1}\left(X \cap V_{i}\right) \cap[-1,1]^{8}$ is the set of common zeros of finitely many real analytic functions on $(-2,2)^{8}$ restricted to $[-1,1]^{8}$. Therefore, it is definable in our o-minimal structure $\mathbf{R}_{\mathrm{an}}$.

Observe that $\theta_{i} \circ \vartheta_{i}$ is real analytic on $(-2,2)^{8}$. Its restriction to $\vartheta_{i}^{-1}\left(U_{i}\right)$ is thus definable. We apply Lemma 2.3 to $\left.\theta_{i} \circ \vartheta_{i}\right|_{\vartheta_{i}^{-1}\left(U_{i}\right)}$ and obtain $M+1$ definable subsets of $\vartheta_{i}^{-1}\left(U_{i}\right)$. Taking their images under $\vartheta_{i}$ gives $U_{i 0}, U_{i 1}, \ldots, U_{i M_{i}}$ with $U_{i}=U_{i 0} \cup \cdots \cup U_{i M_{i}}$. The statement of Lemma 2.3 and the fact that $\vartheta_{i}$ is injective and continuous is what is needed for (i).

Let $X^{\prime}$ be as in part (ii). As before, $\vartheta_{i}^{-1}\left(X^{\prime} \cap V_{i}\right) \cap[-1,1]^{8}$ is a definable set and therefore so is $\vartheta_{i}^{-1}\left(U_{i j}\right) \cap \vartheta_{i}^{-1}\left(X^{\prime} \cap V_{i}\right) \cap[-1,1]^{8}=\vartheta_{i}^{-1}\left(X^{\prime} \cap U_{i j}\right)$. Its image $\theta_{i}\left(X^{\prime} \cap U_{i j}\right)$ under the definable function $\left.\theta_{i} \circ \vartheta_{i}\right|_{[-1,1]^{8}}$ is definable. This shows (ii).

Part (iii) follows since $U_{i}$ is compact and $\theta_{i}$ is continuous.

In order to avoid double indices we rename $U_{i j}$ as $U_{i}$ by increasing, if necessary, the constant $c_{1}$. Of course, we also adjust the $\theta_{i}$ accordingly. For example, in this new notation claim (i) of the preceding lemma states that $\left.\theta_{i}\right|_{U_{i}}$ is either injective or has fibers without isolated points.

We define

$$
W_{i}=\theta_{i}\left(U_{i}\right) \subset \mathbf{R}^{6} .
$$

This is a definable set by part (ii) of the lemma above applied to $X \supset U_{i}$. 
The image of a torsion point of order $N$ in $U_{i}$ lies in $\frac{1}{N} \mathbf{Z}^{6} \cap W_{i}$. For this reason we are interested in the distribution of rational points on $W_{i}$. Below, we will find many such rational points on a fiber of

$$
\begin{aligned}
Z_{i}= & \left\{\left(\xi_{1}, \ldots, \xi_{6}, \alpha, \beta, \gamma, \psi, \omega\right) \in W_{i} \times \mathbf{R}^{5} ;\right. \\
& \left.\alpha \xi_{1}+\beta \xi_{3}+\gamma \xi_{5}=\psi \text { and } \alpha \xi_{2}+\beta \xi_{4}+\gamma \xi_{6}=\omega\right\} \subset \mathbf{R}^{6} \times \mathbf{R}^{5}
\end{aligned}
$$

considered as a family parametrized by $\mathbf{R}^{5}$. We note that the $Z_{i}$ are definable because their definition involve only definable sets and the basic algebraic operations.

The next lemma is the theorem of Pila and Wilkie adapted to our situation.

Lemma 2.5. There exists a positive constant $c_{3}$, depending on the usual data, such that if $1 \leq i \leq c_{1}$ and $y \in \mathbf{R}^{5}$, then

$$
N\left(Y \backslash Y^{\mathrm{alg}}, T\right) \leq c_{3} T^{1 / 12} \text { for all } T \geq 1
$$

where $Y=\left(Z_{i}\right)_{y}$.

Proof. This follows from Theorem 2.2 adapted to our situation.

As we will see below, it is critical that this estimate is uniform in the parameter $y$. We work with the exponent $1 / 12$ for expository reasons; the Theorem of PilaWilkie provides any positive $\epsilon$ at the cost of increasing $c_{3}$.

\subsection{The Galois orbit of a torsion point}

Let $E$ be an elliptic curve defined over a number field $K$. It is well-known that the group of torsion points $E(K)_{\text {tors }}$ of $E(K)$ is finite. By a deep result of Merel its cardinality $\# E(K)_{\text {tors }}$ is bounded from above solely in terms of $[K: \mathbf{Q}]$. In particular, the bound does not depend on the height of $E$. Our method allows us to assume that the height of $E$ is bounded. So the deep uniformity aspect in Merel's work will not play a role here. On the other hand, our argument is quite sensitive in the dependency in $[K: \mathbf{Q}]$ of the bound for \#E $(K)_{\text {tors }}$.

The following result of David is essentially best possible with regard to the degree for an unrestricted elliptic curve.

For a definition and basic properties of the absolute logarithmic Weil height $h$, or just height for short, we refer to Chapter 1.5 in Bombieri and Gubler's book [4].

Theorem 2.6 (David [5]). There exists a positive absolute constant $c_{4}$ with the following property. Let $E$ be an elliptic curve defined over a number field $K$ and let $h_{0} \geq 1$ be a bound for the height of the $j$-invariant of $E$. Then

$$
\# E(K)_{\text {tors }} \leq c_{4} h_{0}[K: \mathbf{Q}] \log (3[K: \mathbf{Q}]) \text {. }
$$

Proof. This follows from [5, Théorème 1.2(i)]. Indeed, torsion points have NéronTate height zero.

Our approach works as long as one has a bound of the form \#E(K) tors $\leq$ $c\left(h_{0}\right)[K: \mathbf{Q}]^{\kappa}$ with fixed $\kappa<3 / 2$ and where $c\left(h_{0}\right)$ is allowed to depend on $h_{0}$. 


\subsection{Torsion points on $X$}

Throughout this subsection we work with a fixed torsion point $P=\left(P_{1}, P_{2}, P_{3}, \lambda\right) \in$ $X(\overline{\mathbf{Q}})$. We will additionally assume

$$
h(\lambda) \leq B
$$

here $B$ is the parameter introduced in beginning of this section. It will be fixed at a later point in the proof and may depend on $X$ but not on $P$. We recall that $\delta, c_{1}, c_{2}, \ldots$ may depend on $B$; but they shall not depend on $P$.

Let $N$ be the order of $P$. For brevity, say $K=F(P) \subset \overline{\mathbf{Q}}$ and $D=[K: F]$. We remark $\lambda \in K \backslash\{0,1\}$. We write $\Sigma$ for the set of embeddings $\sigma: K \rightarrow \mathbf{C}$ that restrict to the identity on $F$. Then $\# \Sigma=D$.

Lemma 2.7. There exist a positive absolute constant $c_{8}$ and $\chi \in \mathbf{Z}^{3} \backslash\{0\}$ with

$$
\max \left\{N,|\chi|^{3}\right\} \leq c_{8} D \log (3 D)
$$

such that $P \in G_{\chi}$.

Proof. The three torsion points $P_{1}, P_{2}, P_{3}$ generate a finite subgroup $\Gamma$ of $\left(\mathcal{E}_{L}\right)_{\lambda}(K)_{\text {tors }}$. Being a finite subgroup of an elliptic curve, $\Gamma$ is isomorphic to $\left(\mathbf{Z} / N^{\prime} \mathbf{Z}\right) \times(\mathbf{Z} / R \mathbf{Z})$ for some positive integers $R \mid N^{\prime}$. Since $\Gamma$ is killed by multiplication by $N$ we find $N^{\prime} \mid N$. But we must have $N^{\prime}=N$ since $P$ has order $N$.

Finding $\chi=(\alpha, \beta, \gamma) \in \mathbf{Z}^{3} \backslash\{0\}$ with $[\alpha]\left(P_{1}\right)+[\beta]\left(P_{2}\right)+[\gamma]\left(P_{3}\right)=0$ on $\left(\mathcal{E}_{L}\right)_{\lambda}$ amounts to finding $(\alpha, \beta, \gamma, *, *) \in \mathbf{Z}^{5} \backslash\{0\}$ in the kernel of a certain matrix

$$
\left[\begin{array}{cccc}
* * & * & N & 0 \\
* * & * & 0 & R
\end{array}\right]
$$

where the entries denoted by $*$ are integers; in the first and second row they lie in $[-N / 2, N / 2]$ and $[-R / 2, R / 2]$, respectively.

We apply Siegel's Lemma as stated in [4, Corollary 2.9.7]. The height of the system (2.2) is at most $c_{5} N R$ with $c_{5}>0$ absolute. Since our system has three independent solutions, there is a solution in $\mathbf{Z}^{5} \backslash\{0\}$ with maximum norm at most $c_{6}(N R)^{1 / 3}$. Forgetting the last two coordinates gives

$$
|\chi| \leq c_{6}(N R)^{1 / 3} \text {. }
$$

On the other hand, we have $N R=\# \Gamma \leq \#\left(\mathcal{E}_{L}\right)_{\lambda}(K)_{\text {tors. }}$. David's result from the last section implies $N R \leq c_{4} h_{0} D \log (3 D)$, here $h_{0}$ is 1 more than the height of the $j$-invariant of $\left(\mathcal{E}_{L}\right)_{\lambda}$. This $j$-invariant equals

$$
j=2^{8} \frac{\left(\lambda^{2}-\lambda+1\right)^{3}}{\lambda^{2}(\lambda-1)^{2}}
$$

by [19, Proposition III 1.7(b)]. Elementary height inequalities imply that $h_{0}$ is bounded in terms of $h(\lambda)$. So $h_{0}$ is bounded in terms of $B$. Hence $N R \leq c_{7} D \log (3 D)$ and in particular $N \leq c_{7} D \log (3 D)$. This is the bound for $N$ in the assertion. We find the bound for $|\chi|^{3}$ by recalling (2.3).

Any embedding $\sigma \in \Sigma$ determines a torsion point $P^{\sigma}=\sigma(P) \in X(\overline{\mathbf{Q}})$. 
Lemma 2.8. For $\delta \in(0,1]$ sufficiently small in terms of $B$ and $F$ there is a positive constant $c_{9} \leq 1$ and an index $1 \leq i_{0} \leq c_{1}$ such that for at least $c_{9} D$ embeddings $\sigma \in \Sigma$ we have

$$
\pi\left(P^{\sigma}\right) \in \Lambda_{\delta} \quad \text { and } \quad P^{\sigma} \in U_{i_{0}}
$$

Proof. A similar statement was given in [11, Lemma 6.2]. Recall $\lambda=\pi(P) \in K$. Let $\delta \in(0,1]$ and let us assume $\sigma(\lambda) \notin \Lambda_{\delta}$ for more than $D / 2$ embeddings $\sigma \in \Sigma$. Then one of

$$
|\sigma(\lambda)|>\delta^{-1}, \quad|\sigma(\lambda)|^{-1}>\delta^{-1}, \quad|1-\sigma(\lambda)|^{-1}>\delta^{-1}
$$

holds for more than $D / 6$ embeddings $\sigma \in \Sigma$.

By elementary height properties we have $h\left(\lambda^{-1}\right)=h(\lambda) \leq B$ and $h\left((1-\lambda)^{-1}\right)=$ $h(1-\lambda) \leq h(\lambda)+\log 2 \leq B+\log 2$. The definition of the height as stated on the bottom of [4, page 16] implies

$$
\begin{aligned}
& h(\lambda)+h\left(\lambda^{-1}\right)+h\left((1-\lambda)^{-1}\right) \\
& \geq \frac{1}{[K: \mathbf{Q}]} \sum_{\sigma: K \rightarrow \mathbf{C}} \log \left(\max \{1,|\sigma(\lambda)|\} \max \left\{1, \frac{1}{|\sigma(\lambda)|}\right\} \max \left\{1, \frac{1}{|1-\sigma(\lambda)|}\right\}\right)
\end{aligned}
$$

here $\sigma$ runs over all embeddings of $K$ into $\mathbf{C}$. We bound $3 B+\log 2 \geq D /(6[K$ : Q]) $\log \left(\delta^{-1}\right)$. But $[K: \mathbf{Q}]=D[F: \mathbf{Q}]$, so $\log \left(\delta^{-1}\right) \leq 6[F: \mathbf{Q}](3 B+\log 2)$.

So if $\delta \in(0,1]$ is sufficiently small with respect to $B$ and $F$ there are at least $D / 2$ embeddings $\sigma \in \Sigma$ satisfying $\sigma(\lambda)=\pi\left(P^{\sigma}\right) \in \Lambda_{\delta}$. Recall that $\left.\pi\right|_{X} ^{-1}\left(\Lambda_{\delta}\right)$ is covered by $U_{1}, \ldots, U_{c_{1}}$. The lemma follows from the Pigeonhole Principle on taking $c_{9}=1 /\left(2 c_{1}\right)$.

We fix $\delta$ and $i$ once and for all as in this lemma and let $\Sigma^{\prime} \subset \Sigma$ denote the subset provided therein. We abbreviate $U=U_{i_{0}}, W=W_{i_{0}}, Z=Z_{i_{0}}$, and $\theta=\theta_{i_{0}}$ from Subsection 2.2. The fact that $i_{0}$ may depend on $P$ will be harmless.

The conjugates $P^{\sigma}$ lie in $U$ for all $\sigma \in \Sigma^{\prime}$. We denote their images under $\theta$ by

$$
\xi^{\sigma}=\left(\xi_{1}^{\sigma}, \ldots, \xi_{6}^{\sigma}\right)=\theta\left(P^{\sigma}\right) \in W
$$

Since $P^{\sigma}$ has order $N$ we have $\xi^{\sigma} \in \frac{1}{N} \mathbf{Z}^{6}$ for the coordinates in terms of the period lattice basis.

Before we continue, let us recapitulate the current situation and also describe how we will proceed. In total there are $D$ conjugates of $P$ over $F$. Of these, a fixed positive proportion lies on the set $U \subset X$. So by Lemma 2.7, the number of conjugates on $U$ is at least of order $N / \log N$. The next lemma is crucial. It states that among the embeddings considered above, at least approximately $N^{1 / 3} / \log N$ yield a $\xi^{\sigma}$ in a fixed fiber of the definable family $Z$ constructed around (2.1). We will show that the number of $\xi^{\sigma}$ equals the number of conjugates $P^{\sigma}$, at least in the most interesting cases. As we have seen above, the $\xi^{\sigma}$ are rational. Their heights turn out to be bounded linearly in terms of $N$. Consequentially, we will 
have found many rational points of bounded height on a fixed fiber of $Z$. But we have no control over the precise fiber containing these rational points; its existence is derived from the Pigeonhole Principle. This is compensated by the fact that the Pila-Wilkie Theorem is uniform over definable families. We then conclude the existence of a semi-algebraic curve inside a fixed fiber of $Z$. Such a curve will lead to a torsion anomalous subvariety of $X$.

Lemma 2.9. There exist a positive constant $c_{12}$, a tuple $y=(\alpha, \beta, \gamma, *, *) \in \mathbf{Z}^{5}$ with $(\alpha, \beta, \gamma) \neq 0$, and a subset $\Sigma^{\prime \prime} \subset \Sigma^{\prime}$ with

$$
\# \Sigma^{\prime \prime} \geq c_{12} \frac{N^{1 / 3}}{\log (3 N)} \quad \text { such that } \quad \xi^{\sigma} \in Z_{y} \quad \text { for all } \quad \sigma \in \Sigma^{\prime \prime}
$$

Proof. Let $\chi=(\alpha, \beta, \gamma)$ be as in Lemma 2.7. Then $P \in G_{\chi}$ and even $P^{\sigma} \in G_{\chi}$ for all $\sigma \in \Sigma$. For $\sigma \in \Sigma^{\prime}$, the period coordinates satisfy

$$
\left(\alpha \xi_{1}^{\sigma}+\beta \xi_{3}^{\sigma}+\gamma \xi_{5}^{\sigma}, \alpha \xi_{2}^{\sigma}+\beta \xi_{4}^{\sigma}+\gamma \xi_{6}^{\sigma}\right) \in \mathbf{Z}^{2}
$$

A simply application of the triangle inequality together with the bound for $\xi_{j}^{\sigma}$ from Lemma 2.4(iii) gives

$$
\left|\alpha \xi_{1}^{\sigma}+\beta \xi_{3}^{\sigma}+\gamma \xi_{5}^{\sigma}\right| \leq 3 c_{2}|\chi|
$$

The same bound holds for $\left|\alpha \xi_{2}^{\sigma}+\beta \xi_{4}^{\sigma}+\gamma \xi_{6}^{\sigma}\right|$. So the number of possibilities for the integral vector $(2.5)$ is at most $\left(6 c_{2}|\chi|+1\right)^{2}$ as $\sigma$ runs over $\Sigma^{\prime}$. Using Lemma 2.7, the number of possibilities is at most $c_{10} D^{2 / 3} \log (3 D)^{2 / 3}$. with

We recall $\# \Sigma^{\prime} \geq c_{9} D$. By the Pigeonhole Principle there is a subset $\Sigma^{\prime \prime} \subset \Sigma^{\prime}$

$$
\# \Sigma^{\prime \prime} \geq \frac{c_{9} D}{c_{10} D^{2 / 3} \log (3 D)^{2 / 3}}=c_{11}\left(\frac{D}{\log (3 D)^{2}}\right)^{1 / 3}
$$

such that (2.5) attains the same value for all $\sigma \in \Sigma^{\prime \prime}$. We use elementary estimates and $N \leq c_{8} D \log (3 D)$ from Lemma 2.7 to conclude

$$
\# \Sigma^{\prime \prime} \geq c_{11}\left(\frac{D \log (3 D)}{\log (3 D)^{3}}\right)^{1 / 3} \geq c_{11}\left(\frac{D \log (3 D)}{\log (3 D \log (3 D))^{3}}\right)^{1 / 3} \geq c_{12} \frac{N^{1 / 3}}{\log (3 N)}
$$

We recall some notation from [6]. There $\operatorname{ker}[N]$ was defined as the kernel of the multiplication by $N$ morphism $[N]: \mathcal{E}_{L}^{3} \rightarrow \mathcal{E}_{L}^{3}$.

Next we find a condition which guarantees that the conjugates of $P$ indeed lead to many rational points $\xi^{\sigma}$. The condition is satisfied if for example $P$ is not inside an anomalous subvariety of $X$. 
Lemma 2.10. Let us assume that $\{P\}$ is an irreducible component of $X \cap \operatorname{ker}[N]$. Then $\left.\theta\right|_{U}: U \rightarrow \mathbf{R}^{6}$ is injective and in particular, $\#\left\{\xi^{\sigma} ; \sigma \in \Sigma^{\prime \prime}\right\}=\# \Sigma^{\prime \prime}$.

Proof. By Lemma 2.4(i) we know that $\left.\theta\right|_{U}$ is either injective or has fibers without isolated points. Say we are in the second case and let us fix $\sigma \in \Sigma^{\prime \prime}$. The fiber of $\theta$ containing any $P^{\sigma}$ also contains an infinite sequence $\left(P_{k}\right)_{k \in \mathbf{N}}$ with $P_{k} \in U \backslash\left\{P^{\sigma}\right\}$ converging to $P^{\sigma}$. Since elliptic logarithms of $P_{k}$ and $P^{\sigma}$ have the same coordinates with respect to a period lattice basis we find $P_{k} \in \operatorname{ker}[N]$. Therefore, $\left\{P^{\sigma}\right\}$ is not an irreducible component of $X \cap \operatorname{ker}[N]$. The same holds true for $\{P\}$ and this contradicts our hypothesis.

We now apply the Theorem of Pila-Wilkie.

Lemma 2.11. Assume $P$ satisfies the hypothesis of Lemma 2.10 and suppose $N$, the order of $P$, is sufficiently large, i.e. $N \geq c_{15}$. There exist $\chi \in \mathbf{Z}^{3} \backslash\{0\}$, an irreducible component $C \subset X \cap G_{\chi}$, and $\sigma \in \Sigma$ with $P^{\sigma} \in C$ such that $\theta(C \cap U)$ contains a connected real semi-algebraic curve.

Proof. Let $y=(\alpha, \beta, \gamma, \psi, \omega) \in \mathbf{Z}^{5} \backslash\{0\}$ and $\Sigma^{\prime \prime}$ be as provided by Lemma 2.9. Say $\sigma \in \Sigma^{\prime \prime}$. Then $P^{\sigma}$ is torsion of order $N$ and we have $\xi^{\sigma} \in \frac{1}{N} \mathbf{Z}^{6}$. On the other hand, $\left|\xi^{\sigma}\right| \leq c_{2}$ by Lemma 2.4(iii). Therefore,

$$
\xi^{\sigma} \in \mathbf{Q}^{6} \quad \text { and } \quad H\left(\xi^{\sigma}\right) \leq c_{13} N \quad \text { with } \quad c_{13}=\max \left\{1, c_{2}\right\} .
$$

We set $T=c_{13} N \geq 1$. By Lemma 2.9 we have $\Sigma^{\prime \prime} \geq c_{14} T^{1 / 3-1 / 6}=c_{14} T^{1 / 6}$. The number of rational points $\xi^{\sigma}$ is thus at least $c_{14} T^{1 / 6}$ by Lemma 2.10. However, the upper bound from Lemma 2.5 gives

$$
N\left(Z_{y} \backslash\left(Z_{y}\right)^{\mathrm{alg}}, T\right) \leq c_{3} T^{1 / 12} .
$$

We may assume that $T=c_{13} N$ is sufficiently large to the end that $c_{14} T^{1 / 6}>$ $c_{3} T^{1 / 12}$. Hence there exists $\sigma \in \Sigma^{\prime \prime}$ with $\xi^{\sigma} \in\left(Z_{y}\right)^{\text {alg }}$. In other words, there is a connected real semi-algebraic set $R$ in $Z_{y}$ of positive dimension that contains $\xi^{\sigma}$.

Any $\xi^{\prime}=\left(\xi_{1}, \ldots, \xi_{6}\right) \in Z_{y}$ satisfies

$$
\alpha \xi_{1}+\beta \xi_{3}+\gamma \xi_{5}=\psi \quad \text { and } \quad \alpha \xi_{2}+\beta \xi_{4}+\gamma \xi_{6}=\omega
$$

By definition, $Z_{y} \subset W=\theta(U)$. So there is $Q \in U$ with $\theta(Q)=\xi^{\prime}$. The linear relations imply $Q \in G_{\chi}$ with $\chi=(\alpha, \beta, \gamma)$. We conclude $Z_{y} \subset \theta\left(U \cap G_{\chi}\right)$. Let $X \cap G_{\chi}=C_{1} \cup \cdots \cup C_{r}$ be the decomposition into irreducible components. So $Z_{y} \subset \bigcup_{k} \theta\left(C_{k} \cap U\right)$.

Since $Z_{y}$ contains a connected real semi-algebraic set of positive dimension that passes through $\xi^{\sigma}$, it is reasonable to expect some $\theta\left(C_{k} \cap U\right)$ to do the same. Let us now prove this fact. By [21, Proposition 3.2, page 100] there is a continuous semi-algebraic function $\gamma:[0,1] \rightarrow Z_{y}$ with $\gamma(0)=\xi^{\sigma}$ and $\gamma(1) \neq \gamma(0)$. Each $\theta\left(C_{k} \cap U\right)$ is definable by Lemma 2.4(ii). The pre-images $I_{k}=\gamma^{-1}\left(\theta\left(C_{k} \cap\right.\right.$ 
$U)) \subset \mathbf{R}$ are definable and their union is $[0,1]$. Recall that $U$ is compact. So each $I_{k}$ is closed because $\theta\left(C_{k} \cap U\right) \subset \mathbf{R}^{6}$ is closed. By property (v) of an ominimal structure, each $I_{k}$ is a finite union of closed intervals. So there is $k$ and $t \in(0,1]$ such that $I_{k}$ has $[0, t]$ as a connected component. We may choose $k$ such that $t$ is maximal. So $\left.\gamma\right|_{[0, t]}$ maps to $\theta(C \cap U)$ with $C=C_{k}$; in particular, $\xi^{\sigma} \in \theta(C \cap U)$. What if $\left.\gamma\right|_{[0, t]}$ is constant? Then $t<1$ because $\gamma(1) \neq \gamma(0)$. By a similar argument as above, the interval $[t, 1]$ can be covered by pre-images which are themselves finite unions of closed intervals. From this we deduce a contradiction to the maximality of $t$. So $\left.\gamma\right|_{[0, t]}$ is non-constant. Its image is a connected real semialgebraic curve which is completely contained in $\theta(C \cap U)$.

This implies the second assertion of the lemma. It also shows that $\xi^{\sigma}=\theta\left(P^{\prime}\right)$ for some $P^{\prime} \in C \cap U$. But recall that $\left.\theta\right|_{U}$ is injective by Lemma 2.10 and $\theta\left(P^{\sigma}\right)=$ $\xi^{\sigma}$. Therefore, $P^{\sigma}=P^{\prime} \in C$.

Lemma 2.12. Let $C \subset \mathcal{E}_{L}^{3}$ be an irreducible algebraic curve such that $\theta(U \cap C)$ contains a connected real semi-algebraic curve.

(i) If $\left.\pi\right|_{C}: C \rightarrow Y(2)$ is dominant there exist independent $\chi^{\prime}, \chi^{\prime \prime} \in \mathbf{Z}^{3}$ with $C \subset G_{\chi^{\prime}} \cap G_{\chi^{\prime \prime}}$

(ii) If $\left.\pi\right|_{C}: C \rightarrow Y(2)$ is not dominant, then it is constant and $C$ is the translate of an algebraic subgroup of $\left(\mathcal{E}_{L}^{3}\right)_{\pi(C)}$.

Proof. Part (i) follows from Bertrand's [2, Théorème 5] applied to the three possible projections of $C$ onto $\mathcal{E}_{L}^{2}$. Alternatively, we can also refer to Masser and Zannier's [9, Appendix A].

Part (ii) is a consequence of Ax's [1, Theorem 3] for a fixed Abelian variety.

\subsection{Proof of Proposition 2.1}

We begin by fixing the parameter $B$ used above.

By [6, Theorem 1.3(ii)] there exists $B \geq 1$, depending on $X$, with $h(\pi(P)) \leq$ $B$ for all torsion points $P \in X^{\star} \cap X(\overline{\mathbf{Q}})$.

Let $P \in X^{\star}$ be a torsion point of order $N$ and set $\lambda=\pi(P)$.

The Zariski closed set $\operatorname{ker}[N]$ is equidimensional of dimension 1 by [6, Lemma 2.5]. So $\{P\}$ is an irreducible component of the intersection $X \cap \operatorname{ker}[N]$. We can deduce two things. First, using the fact that $X$ and $\operatorname{ker}[N]$ are defined over $\overline{\mathbf{Q}}$ we find that $P$ is algebraic, i.e. $P \in X(\overline{\mathbf{Q}})$. Second, $h(\lambda) \leq B$. So $P$ is as in Subsection 2.4.

After omitting finitely many $P$ we may suppose that $N$ is sufficiently large; for example $N \geq c_{15}$, the constant from Lemma 2.11. We remark that $P$ satisfies the hypothesis of this lemma.

We will prove part (ii) first. So we shall additionally assume that $\left(\mathcal{E}_{L}\right)_{\lambda}$ has complex multiplication. The $j$-invariant $J$ of the elliptic curve $\left(\mathcal{E}_{L}\right)_{\lambda}$ is given by (2.4). By basic height properties and $h(\lambda) \leq B$, we find that $h(J)$ is bounded from above independently of $P$. A result of Poonen [15] states that the set of $j$-invariants of bounded height coming from elliptic curves with complex multiplication is finite. 
So there are only finitely many possibilities for $J$. By (2.4) the same holds true for $\lambda$.

We now prove part (i). We now assume in addition $P \in X^{\text {ta }} \subset X^{\star}$.

We have already assumed $N$ to be large; this will lead to a contradiction as follows. Let $\chi \in \mathbf{Z}^{3} \backslash\{0\}$ and $C \subset X \cap G_{\chi}$ be as in Lemma 2.11. Then $C \neq X$ because otherwise $X \subset G_{\chi}$ would imply $X^{\text {ta }}=\emptyset$. So $\operatorname{dim} C \leq 1$. General intersection theory implies $\operatorname{dim} C \geq \operatorname{dim} X-1$. Hence $C$ is an algebraic curve defined over $\overline{\mathbf{Q}}$. Recall that $P^{\sigma}$ lies on $C$ for some $\sigma \in \Sigma$. We split up into cases regarding whether $\left.\pi\right|_{C}: C \rightarrow Y(2)$ is dominant or not.

First we assume $\left.\pi\right|_{C}$ is dominant. By Lemma 2.12(i) the algebraic curve $C$ lies in $G_{\chi^{\prime}} \cap G_{\chi^{\prime \prime}}$ for independent $\chi^{\prime}, \chi^{\prime \prime} \in \mathbf{Z}^{3}$. But for an appropriate conjugate $C^{\prime}$ of $C$ we have $P \in C^{\prime}$ and $C^{\prime} \subset G_{\chi^{\prime}} \cap G_{\chi^{\prime \prime}}$. Therefore, $C^{\prime}$ is torsion anomalous which contradicts $P \in X^{\mathrm{ta}}$.

Now say $\left.\pi\right|_{C}$ is not dominant. This means that $C$ is contained in a single fiber of $\mathcal{E}_{L}^{3} \rightarrow Y(2)$. We know from Lemma 2.12(ii) that $C$ is a translate of an algebraic subgroup of a fiber of $\mathcal{E}_{L}^{3}$. But $C$ contains $P^{\sigma}$, which is torsion. So $C$ is the translate of an algebraic subgroup by a torsion point. Conjugating, we find that $P$ is on an algebraic curve $C^{\prime}$ which is the translate of an algebraic subgroup of $\left(\mathcal{E}_{L}^{3}\right)_{\lambda}$ by a torsion point.

If $\left(\mathcal{E}_{L}\right)_{\lambda}$ does not have complex multiplication then $C^{\prime} \subset G_{\chi^{\prime}} \cap G_{\chi^{\prime \prime}}$ for independent $\chi^{\prime}, \chi^{\prime \prime} \in \mathbf{Z}^{3}$. This means that $C^{\prime}$ is a torsion anomalous subvariety of $X$ as in part (i) of the definition. But $P \in C^{\prime}$, contradicting our hypothesis $P \in X^{\text {ta }}$.

Finally, suppose $\left(\mathcal{E}_{L}\right)_{\lambda}$ has complex multiplication. Then $C^{\prime}$ is a torsion anomalous subvariety as in part (iii) of the definition. As above we arrive at a contradiction.

\section{Torsion anomalous subvarieties}

The results in this section are formulated using the Weierstrass family of elliptic curves. Recall that the base $S$ is the algebraic surface given by (1.2). The fiber above $(a, b) \in S$ is an elliptic curve with $j$-invariant $j(a, b)=2^{8} 3^{3} a^{3} /\left(4 a^{3}+27 b^{2}\right)$. We regard $j: S \rightarrow \mathbf{A}^{1}$ as a morphism.

Recall that $\mathcal{E}^{3}$ is a five-dimensional non-singular irreducible variety. By abuse of notation, $\pi$ denotes both structure morphisms $\mathcal{E} \rightarrow S$ and $\mathcal{E}^{3} \rightarrow S$. Both are proper morphisms. It is straightforward to check that the 123 -surface $X$ is irreducible.

In Section 2 we defined torsion anomalous subvarieties of an irreducible algebraic surface in $\mathcal{E}_{L}^{3}$. The analog definition for a surface in $\mathcal{E}^{3}$ is somewhat more involved. This is due to the fact that fibers of $\mathcal{E}^{3} \rightarrow S$ are isomorphic along algebraic curves in $S$ where $j$ is constant. Before coming to the definition we state an elementary lemma which is used through this section. It enables us to pass from the Weierstrass to the Tate model of an elliptic curve.

If $K$ is a field then $K^{\times}=K \backslash\{0\}$. 
Lemma 3.1. Let $K$ be a field of characteristic not equal to 2 or 3. Say we are given two elliptic curves

$$
\begin{gathered}
E: y^{2}=x^{3}+a x+b \quad \text { and } \\
E^{\prime}: y^{2}+x y=x^{3}+a^{\prime} x+b^{\prime}
\end{gathered}
$$

with $a, b, a^{\prime}, b^{\prime} \in K$ that are isomorphic over $K$. Then there exists $w \in K^{\times}$such that

$$
(x, y) \mapsto\left(w^{2} x-\frac{1}{12}, w^{3} y-\frac{1}{2} w^{2} x+\frac{1}{24}\right)
$$

determines an isomorphism $E \rightarrow E^{\prime}$ with

$$
w^{4} a=a^{\prime}-\frac{1}{48} \quad \text { and } \quad w^{6} b=-\frac{1}{12} a^{\prime}+b^{\prime}+\frac{1}{864} .
$$

Proof. This follows from the basic theory of elliptic curves [19].

Now we come to the auxiliary construction needed for the definition of torsion anomalous subvarieties. Let $A \subset \mathcal{E}_{L}^{3}$ be an irreducible closed subvariety such that $\left.j \circ \pi\right|_{A}$ is constant with value $J \in \mathbf{C}$. Then $\pi(A)$ is either a point or an irreducible algebraic curve.

We assume the latter for the moment and set $C=\pi(A)$. We take the coordinates $a$ and $b$ of $S$ as elements in the function field $\mathbf{C}(C)$ of $C$. Then $4 a^{3}(J-$ $1728)+27 b^{2} J=0$. So $\mathbf{C}(a, b)$ is a rational function field generated by some $t \in \mathbf{C}(C)$. We may assume

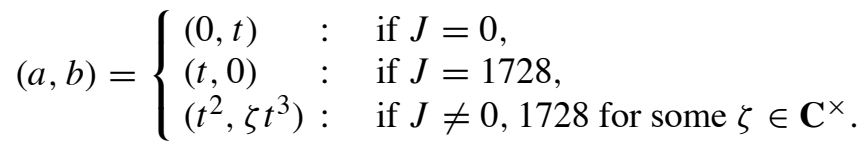

The equation $y^{2}=x^{3}+a x+b$ defines an elliptic curve $E$ over $\mathbf{C}(t)$. By the basic theory, there is an elliptic curve $E^{\prime}$ as in (3.1) with $a^{\prime}, b^{\prime} \in \mathbf{C}$ and $j$-invariant $J$. Now $E$ and $E^{\prime}$ are isomorphic over an algebraic closure $\overline{\mathbf{C}(t)}$ of $\mathbf{C}(t)$ as they share a common $j$-invariant. Lemma 3.1 provides $w \in \overline{\mathbf{C}(t)}^{\times}$and an isomorphism between $E$ and $E^{\prime}$. We regard $E^{\prime}$ as an elliptic curve defined over $\mathbf{C}$. The isomorphism may be taken as an algebraic map on $\pi^{-1}(C)$ with image $E^{\prime 3}$. We let $A^{\prime}$ denote the Zariski closure of the image of $A$ in $E^{\prime 3}$.

If $\pi(A)$ is a point, then we take $A^{\prime}=A$ regarded as a subvariety of the Abelian variety $E^{\prime 3}=\pi^{-1}(\pi(A))$.

Let $A$ be an arbitrary irreducible closed subvariety of an algebraic surface in $\mathcal{E}^{3}$. Then $A$ is called torsion anomalous with respect to the given surface

(i) if $\operatorname{dim} A=1$ and two independent integral relations hold on $A$,

(ii) or if $\operatorname{dim} A=2$ and one non-trivial integral relation holds on $A$, 
(iii) or if $\operatorname{dim} A \geq 1$ and $\left.j \circ \pi\right|_{A}$ is constant and equal to the $j$-invariant of an elliptic curve with complex multiplication such that, in the notation above, $A^{\prime}$ is an irreducible component of an algebraic subgroup of $E^{\prime 3}$.

Proposition 2.1 contained a finiteness statement on the torsion points outside the torsion anomalous locus of a surface in $\mathcal{E}_{L}^{3}$. The torsion anomalous subvarieties of the 123-surface will cause no problems.

Proposition 3.2. The 123-surface contains no torsion anomalous subvarieties.

\subsection{Constant $j$-invariant}

As a warm-up for the proof of Theorem 1.1 we show the following weaker version. An algebraic curve in the 123-surface on which $j$ is constant contains only finitely many torsion points. We will use this statement in the proof of Proposition 3.2.

Lemma 3.3. Let $A \subset X$ be an irreducible closed subvariety such that $\left.j \circ \pi\right|_{A}$ is constant. Then A contains only finitely many torsion points and A is not a torsion anomalous subvariety as in part (iii) of the definition.

Proof. We may assume $\operatorname{dim} A \geq 1$. We remark that $A$ and $\pi(A)=C$ are algebraic curves since $\left.\pi\right|_{X}$ is dominant and has finite fibers.

Let $J \in \mathbf{C}$ be said $j$-invariant. We let $w, t, A^{\prime}$, and $E^{\prime}$ be as in the auxiliary construction before the definition of anomalous subvarieties. We also consider $a$ and $b$ as elements in $\mathbf{C}(t)$.

We note that $w \notin \mathbf{C}$, indeed, otherwise $a, b$ would be constant as well by (3.2). Using (3.3) we find that $1+a+b \in \mathbf{C}(t)$ has odd degree. Therefore, there is a non-trivial valuation ord of $\mathbf{C}(t)$ with ord $(1+a+b)$ positive and odd. Using (3.3) again one finds ord $(t)=0$. Because $a^{\prime}, b^{\prime} \in \mathbf{C}$ we can deduce $\operatorname{ord}(w)=0$ from (3.2). Therefore, $\mathbf{C}(w, t) / \mathbf{C}(t)$ is unramified above ord. Since $\{(1,1,1),(8,2,1),(27,3,1)\}$ is linearly dependent we must have ord $(8+2 a+b)=$ 0 or ord $(27+3 a+b)=0$. For simplicity say the former holds; the argument below is readily modified in the latter case. We set $K=\mathbf{C}\left(y_{2}, w, t\right)$, then $K / \mathbf{C}(t)$ is unramified above ord. We extend this valuation to $K$ and note that $K\left(y_{1}\right) / K$ is ramified. Because $y_{1}^{2} \in K$ the extension $K\left(y_{1}\right) / K$ is of degree 2 and there is an automorphism $\sigma$ of $K\left(y_{1}\right) / K$ with $\sigma\left(y_{1}\right)=-y_{1}$.

For $i \in\{1,2,3\}$ we have a point $\left(i, y_{i}\right) \in E(\overline{\mathbf{C}(t)})$. Its image in $E^{\prime}(\overline{\mathbf{C}(t)})$ under the isomorphism coming from Lemma 3.1 is

$$
\left(w^{2} i-\frac{1}{12}, w^{3} y_{i}-\frac{1}{2} w^{2} i+\frac{1}{24}\right) .
$$

We may regard $w, y_{1,2,3}$ as rational functions on a ramified cover of $A$. The three points (3.4) determine a rational map from this cover to $E^{\prime 3}$. Then $A^{\prime}$ is the Zariski closure of its image. If $A$ contains infinitely torsion points then so does $A^{\prime}$. We use the Manin-Mumford Conjecture for Abelian varieties, a result first proved by Raynaud [16]. It implies that $A^{\prime}$ is an irreducible component of an algebraic subgroup 
of $E^{\prime 3}$. In particular, there are endomorphisms $\alpha, \beta$ of $E^{\prime}$, not both zero, such that $\alpha\left(P_{1}\right)=\beta\left(P_{2}\right)$ for all $\left(P_{1}, P_{2}, P_{3}\right) \in A^{\prime}$. This relation continues to hold generically, i.e. $\alpha\left(P_{1}^{\prime}\right)=\beta\left(P_{2}^{\prime}\right)$ with $P_{i}^{\prime}=\left(w^{2} i-1 / 12, w^{3} y_{i}-w^{2} i / 2+1 / 24\right) \in E(\overline{\mathbf{C}(t)})$. Because $\sigma$ commutes with all endomorphisms of $E^{\prime}$, which are defined over $\mathbf{C}$, we get

$$
\begin{aligned}
-\beta\left(P_{2}^{\prime}\right)=-\alpha\left(P_{1}^{\prime}\right) & =\alpha\left(w^{2}-1 / 12,-w^{3} y_{1}-w^{2} / 2+1 / 24\right)=\alpha\left(P_{1}^{\prime}\right)^{\sigma} \\
& =\beta\left(P_{2}^{\prime}\right)^{\sigma}=\beta\left(P_{2}^{\prime}\right) .
\end{aligned}
$$

Therefore, $2 \beta\left(P_{2}^{\prime}\right)=0$. So one of $P_{1}^{\prime}, P_{2}^{\prime} \in E^{\prime}(\overline{\mathbf{C}(t)})$ is a torsion point. But these torsion points are defined over $\mathbf{C}$ and hence $w \in \mathbf{C}$. This contradicts the fact that $w$ is non-constant.

So $A$ contains only finitely many torsion points. Because an algebraic subgroup of an Abelian variety contains a Zariski dense set of torsion points we also conclude that $A$ is not torsion anomalous as in part (iii) of the definition.

\subsection{Tate curves}

In this subsection we collect some basic facts on Tate curves. A general reference is Chapter V of Silverman's book [18] or Roquette's book [17].

Let $K_{v}$ be a field, complete with respect to a discrete valuation $v: K_{v} \rightarrow$ $\mathbf{Z} \cup\{+\infty\}$ which we assume to be surjective. If $q \in K_{v}^{\times}$with $v(q)>0$ then the Weierstrass equation

$$
y^{2}+x y=x^{3}+a_{4}(q) x+a_{6}(q)
$$

defines the Tate curve $E_{q}$ where

$$
a_{4}=-\sum_{n \geq 1} \frac{n^{3} q^{n}}{1-q^{n}} \quad \text { and } \quad a_{6}=-\frac{1}{12} \sum_{n \geq 1} \frac{\left(5 n^{3}+7 n^{5}\right) q^{n}}{1-q^{n}}
$$

converge in $K_{v}, c f$. [18, Theorem V 3.1]. By this theorem and Remark V 3.1.2 ibid., $c f$. Roquette's work cited above, there exists a surjective homomorphism of groups

$$
\phi: K_{v}^{\times} \rightarrow E_{q}\left(K_{v}\right)
$$

with kernel $q^{\mathbf{Z}}$, the infinite cyclic subgroup of $K_{v}^{\times}$generated by $q$.

We follow a convenient convention and represent points of $E_{q}\left(K_{v}\right) \backslash\{0\}$ using affine coordinates.

Equation (3.5) has coefficients in the ring of integers of $K_{v}$ and is minimal. Let $L$ be the residue field of $K_{v}$. The reduction $\widetilde{E_{q}}$ of $E_{q}$ is an irreducible projective curve defined over $L$. We have the reduction map red $: E_{q}\left(K_{v}\right) \rightarrow \widetilde{E_{q}}(L)$. The set of non-singular points of $\widetilde{E_{q}}(L)$ carries a natural Abelian group structure. We define

$$
E_{q}\left(K_{v}\right)_{0}=\left\{P \in E_{q}\left(K_{v}\right) ; \operatorname{red}(P) \text { is non-singular on } \widetilde{E_{q}}\right\} .
$$


This is a subgroup of finite index of $E_{q}\left(K_{v}\right)$ and red $\left.\right|_{E_{q}\left(K_{v}\right)_{0}}$ is a homomorphism of groups.

The Tate uniformization $\phi$ lets us do calculations explicitly on Tate curves.

Lemma 3.4. Let $P \in E_{q}\left(K_{v}\right)_{0} \backslash\{0\}$. There is a unique $\tilde{u} \in K_{v}$ with $v(\tilde{u})=0$ and $\phi(\tilde{u})=P$. Moreover, if $u \in L$ is the reduction of $\tilde{u}$ then $u \neq 0$ and

(i) either $u=1$ and $\operatorname{red}(P)=0$,

(ii) or $u \neq 1$ and $\operatorname{red}(P)=\left(\frac{u}{(1-u)^{2}}, *\right) \neq 0$.

Proof. There is precisely one $\tilde{u} \in K_{v}^{\times} \backslash q^{\mathbf{Z}}$ with $0 \leq v(\tilde{u})<v(q)$ and $\phi(\tilde{u})=$ $(x, y)=P$. By [18, Lemma V 4.1.1] we have $v(x) \leq 0$ because $P \in E_{q}\left(K_{v}\right)_{0}$; we remark that the proof of this lemma involves only formal properties of the valuation on $K_{v}$ and hence holds for any valued field.

The homomorphism $\phi$ is explicitly given in [18, Theorem V 3.1] as

$$
\phi(\tilde{u})=\left(\sum_{n \in \mathbf{Z}} \frac{q^{n} \tilde{u}}{\left(1-q^{n} \tilde{u}\right)^{2}}-2 \sum_{n \geq 1} \frac{n q^{n}}{1-q^{n}}, \sum_{n \in \mathbf{Z}} \frac{q^{2 n} \tilde{u}^{2}}{\left(1-q^{n} \tilde{u}\right)^{3}}+\sum_{n \geq 1} \frac{n q^{n}}{1-q^{n}}\right)
$$

because $\tilde{u} \notin q^{\mathbf{Z}}$. All terms in the sum for $x$ have positive valuation except possibly $\frac{q^{n} \tilde{u}}{\left(1-q^{n} \tilde{u}\right)^{2}}$ for $n=0$. A similar remark holds for $y$. We can write

$$
P=\left(\frac{\tilde{u}}{(1-\tilde{u})^{2}}+x^{\prime}, \frac{\tilde{u}^{2}}{(1-\tilde{u})^{3}}+y^{\prime}\right) \quad \text { with } \quad v\left(x^{\prime}\right)>0 \quad \text { and } \quad v\left(y^{\prime}\right)>0 .
$$

Since $v(x) \leq 0$ we must have $v(\tilde{u}) \leq 2 v(1-\tilde{u})$. This inequality implies $v(\tilde{u})=0$. The reduction $u$ of $\tilde{u}$ is thus non-zero in the residue field $L$.

If $v(1-\tilde{u})>0$, then $u=1$ in $L$. The orders satisfy

$$
v(x)=-2 v(1-\tilde{u}) \text { and } \quad v(y)=-3 v(1-\tilde{u}) .
$$

In particular, $y \neq 0$ and in projective coordinates we have $P=[x / y: 1: 1 / y]$ with $v(x / y)=v(1-\tilde{u})>0$ and $v(1 / y)=3 v(1-\tilde{u})>0$. Therefore, $\operatorname{red}(P)=0$ and we are in case (i).

On the other hand, if $v(1-\tilde{u}) \leq 0$, then $v(1-\tilde{u})=0$ and so $u \neq 1$. From (3.6) we see that $x$ reduces to $u /(1-u)^{2}$ in the $L$. We are in case (ii).

\subsection{Function fields}

Let $K$ be the function field of an irreducible algebraic curve defined over $\mathbf{C}$. Let $a, b \in K$ with $4 a^{3}+27 b^{2} \neq 0$. Then

$$
y^{2}=x^{3}+a x+b
$$

determines an elliptic curve $E$ defined over $K$. 
After replacing $K$ by a finite extension we have points

$$
P_{1}=(1, *) \in E(K), \quad P_{2}=(2, *) \in E(K), \quad \text { and } \quad P_{3}=(3, *) \in E(K) .
$$

The choice of sign of the second coordinate will be irrelevant. After again passing to a finite extension of $K$ we may assume that $E$ has either good or multiplicative reduction at all places of $K$. Multiplicative reduction is automatically split because the residue field $\mathbf{C}$ is algebraically closed.

For any place $v$ of $K$ we let $K_{v}$ denote the completion of $K$ with respect to $v$. We identify $v$ with the corresponding surjective valuation $K_{v} \rightarrow \mathbf{Z} \cup\{+\infty\}$. We define a finite (possibly empty) set

$$
S=\text { \{places of } K \text { where } E \text { has bad reduction }\} .
$$

If $v \in S$, then $E$ is isomorphic over $K_{v}$ to the Tate curve $E_{q_{v}}$ for some $q_{v} \in K_{v}^{\times}$ with $v\left(q_{v}\right)>0$. Let $f_{v}: E \rightarrow E_{q_{v}}$ be an isomorphism as in Lemma 3.1. If $v \notin S$, then $E$ is isomorphic over $K_{v}$ to an elliptic curve $E_{v}$ given by the equation $y^{2}+x y=x^{3}+a^{\prime} x+b^{\prime}$ with $a^{\prime}, b^{\prime}$ integers in $K_{v}$ and with good reduction. Let $f_{v}: E \rightarrow E_{v}$ be an isomorphism given by said lemma. To unify notation we sometimes write $E_{v}=E_{q_{v}}$ if $v \in S$.

Lemma 3.5. Let $v \in S$ and $i, j \in\{1,2,3\}$ with $i \neq j$. If $f_{v}\left(P_{i}\right) \notin E_{v}\left(K_{v}\right)_{0}$, then

$$
\operatorname{red} f_{v}\left(P_{j}\right)=\left(\frac{1}{12}\left(\frac{j}{i}-1\right), *\right) \in \widetilde{E_{v}}(\mathbf{C}) \quad \text { and } \quad f_{v}\left(P_{j}\right) \in E_{v}\left(K_{v}\right)_{0} .
$$

Proof. The isomorphism $f_{v}$ is given on the affine part of $E$ by

$$
(x, y) \mapsto\left(w^{2} x-\frac{1}{12}, *\right) .
$$

for some $w \in K_{v}^{\times}$. The reduction $\widetilde{E_{v}}$ is determined by the Weierstrass equation $y^{2}+x y=x^{3}$ and $(0,0)$ is its only singular point. By hypothesis, $f_{v}\left(P_{i}\right)$ reduces to $(0,0)$. Therefore, $v\left(w^{2} i-1 / 12\right)>0$. Since $i \neq 0$ we find $v\left(w^{2}-1 /(12 i)\right)>0$. In other words, $w^{2}$ reduces to $1 /(12 i)$ at $v$. So $w^{2} j-1 / 12$ reduces to $(j / i-1) / 12$ at $v$ and this is the first coordinate of red $f_{v}\left(P_{j}\right)$. Finally, because $i \neq j$ we have $f_{v}\left(P_{j}\right) \in E_{v}\left(K_{v}\right)_{0}$.

For $i \in\{1,2,3\}$ we define the finite (possibly empty) set

$$
S_{i}=\left\{v \in S ; \quad f_{v}\left(P_{i}\right) \notin E_{v}\left(K_{v}\right)_{0}\right\} .
$$

For a finite sequence $P, \ldots, Q \in E(K)$ we set $\rho(P, \ldots, Q)$ to be the rank of the Z-submodule of $E(K)$ generated by $P, \ldots, Q$.

Lemma 3.6. Suppose $\rho\left(P_{1}, P_{2}, P_{3}\right) \leq 1$. Then $S_{1}=S_{2}=\emptyset$. 
Proof. Say $i \in\{1,2\}$. Assuming the existence of $v \in S_{i}$ we will eventually arrive at a contradiction.

Let us fix $j$ and $k$ with $\{i, j, k\}=\{1,2,3\}$ and $j<k$. By Lemma 3.5 we find

$$
\operatorname{red} f_{v}\left(P_{j}\right)=\left(\frac{1}{12}\left(\frac{j}{i}-1\right), *\right) \neq 0, \quad \operatorname{red} f_{v}\left(P_{k}\right)=\left(\frac{1}{12}\left(\frac{k}{i}-1\right), *\right) \neq 0
$$

and $f_{v}\left(P_{j}\right), f_{v}\left(P_{k}\right) \in E_{v}\left(K_{v}\right)_{0} \backslash\{0\}$.

We apply Lemma 3.4 to $f_{v}\left(P_{j}\right)$ and $f_{v}\left(P_{k}\right)$ and obtain elements $\tilde{u} \in K_{v}$ and $\tilde{u}^{\prime} \in K_{v}$, respectively. We are in case (ii) of said lemma, so $u \neq 1$ and $u^{\prime} \neq 1$ for the reductions of $\tilde{u}$ and $\tilde{u}^{\prime}$, respectively. These reductions satisfy

$$
\frac{u}{(1-u)^{2}}=\frac{1}{12}\left(\frac{j}{i}-1\right), \quad \text { and } \quad \frac{u^{\prime}}{\left(1-u^{\prime}\right)^{2}}=\frac{1}{12}\left(\frac{k}{i}-1\right) .
$$

Since $\rho\left(P_{1}, P_{2}, P_{3}\right) \leq 1$ we have $\rho\left(P_{j}, P_{k}\right) \leq 1$. So there are $M, N \in \mathbf{Z}$, not both zero, with $[M]\left(P_{j}\right)=[N]\left(P_{k}\right)$. Using the Tate uniformization, this relation reads $\phi\left(\tilde{u}^{M}\right)=[M]\left(f_{v}\left(P_{j}\right)\right)=[N]\left(f_{v}\left(P_{k}\right)\right)=\phi\left(\tilde{u}^{\prime N}\right)$. So $\tilde{u}^{M} \tilde{u}^{\prime-N} \in q^{\mathbf{Z}}$. Since $\tilde{u}$ and $\tilde{u}^{\prime}$ have valuation zero, we find $\tilde{u}^{M}=\tilde{u}^{\prime N}$ and in particular, $u^{M}=u^{\prime N}$.

The contradiction now follows by simply evaluating $u$ and $u^{\prime}$ in the two possible cases $i=1,2$ using (3.7). Rewriting these identities gives

$$
u^{2}+2 \frac{5 i+j}{i-j} u+1=0 \text { and } u^{\prime 2}+2 \frac{5 i+k}{i-k} u^{\prime}+1=0
$$

with solutions

$$
\left(u, u^{\prime}\right)= \begin{cases}(7 \pm 4 \sqrt{3}, 4 \pm \sqrt{15}) & : \text { if }(i, j, k)=(1,2,3) \\ (-11 \pm 2 \sqrt{30}, 13 \pm 2 \sqrt{42}) & : \text { if }(i, j, k)=(2,1,3)\end{cases}
$$

In both cases $u, u^{\prime}$ are algebraic units with $\mathbf{Q}(u) \cap \mathbf{Q}\left(u^{\prime}\right)=\mathbf{Q}$. Hence $u^{M}=$ $u^{\prime N} \in\{ \pm 1\}$, the algebraic units of $\mathbf{Q}$. So one among $u, u^{\prime}$ is a root of unity. This is impossible for the totally real $u$ and $u^{\prime}$; the lemma follows.

One can go a bit further and also show $S_{3}=\emptyset$. But this will not be necessary.

If $v$ is any place of $K$, then $\lambda_{v}$ denotes the Néron local height on any elliptic curve over $K_{v}, c f$. Chapter VI [18]. It does not depend on the choice of a model of the elliptic curve. For a place $v$ of bad reduction we will use the Tate curve $E_{q_{v}}=$ $E_{v}$ to calculate $\lambda_{v}$. There is an explicit formula for $\lambda_{v}$ restricted to $E_{v}\left(K_{v}\right)_{0} \backslash\{0\}$ given by [18, Theorem VI 4.1]. We can use it to handle $\lambda_{v}\left(P_{1}\right)$ and $\lambda_{v}\left(P_{2}\right)$ because $S_{1}=S_{2}=\emptyset$.

Lemma 3.7. Suppose $\rho\left(P_{1}, P_{2}, P_{3}\right) \leq 1$. If $v$ is any place of $K$, then

$$
\lambda_{v}\left(P_{1}\right)=\lambda_{v}\left(P_{2}\right) .
$$


Proof. Let $v$ be any place of $K$. Recall that $f_{v}: E \rightarrow E_{v}$ is an isomorphism of elliptic curves over $K$. By Lemma 3.6 the points $f_{v}\left(P_{1}\right)$ and $f_{v}\left(P_{2}\right)$ reduce to a non-singular point.

Since $P_{1,2} \neq 0$ we may use Theorem VI 4.1 to evaluate

$$
\begin{aligned}
& \lambda_{v}\left(P_{1}\right)=\lambda_{v}\left(f_{v}\left(P_{1}\right)\right)=\frac{1}{2} \max \left\{0,-v\left(x_{1}\right)\right\}+\frac{1}{12} v\left(\Delta_{v}\right) \quad \text { and } \\
& \lambda_{v}\left(P_{2}\right)=\lambda_{v}\left(f_{v}\left(P_{2}\right)\right)=\frac{1}{2} \max \left\{0,-v\left(x_{2}\right)\right\}+\frac{1}{12} v\left(\Delta_{v}\right)
\end{aligned}
$$

where $x_{1}$ and $x_{2}$ are the first coordinates of $f_{v}\left(P_{1}\right)$ and $f_{v}\left(P_{2}\right)$, respectively, and $\Delta_{v}$ is the local discriminant of $E_{v}$. We remark that $x_{1}$ and $x_{2}$ depend on $v$.

By Lemma 3.1, the isomorphism $f_{v}$ is determined by some $w \in K_{v}^{\times}$. So

$$
x_{1}=w^{2}-\frac{1}{12} \quad \text { and } \quad x_{2}=2 w^{2}-\frac{1}{12} .
$$

We split up into two cases.

First, let us suppose $v(w) \geq 0$. Then $v\left(x_{1}\right) \geq 0$ and $v\left(x_{2}\right) \geq 0$ by the ultrametric triangle inequality. So we have

$$
\lambda_{v}\left(P_{1}\right)=\lambda_{v}\left(P_{2}\right)=\frac{1}{12} v\left(\Delta_{v}\right) .
$$

Second, we assume $v(w)<0$. In this case the ultrametric triangle inequality yields $v\left(x_{1}\right)=v\left(x_{2}\right)=v\left(w^{2}\right)$. Therefore,

$$
\lambda_{v}\left(P_{1}\right)=\lambda_{v}\left(P_{2}\right)=-\frac{1}{2} v\left(w^{2}\right)+\frac{1}{12} v\left(\Delta_{v}\right) .
$$

Now we will show that $E$ has good reduction everywhere under the hypothesis of the previous lemma. This is done by a global argument using local data from the last lemma.

The Néron-Tate or canonical height is defined for $P \in E(K) \backslash\{0\}$ as $\hat{h}(P)=$ $\sum_{v} \lambda_{v}(P)$ where the sum runs over all places of $K$; for $P=0$ we set $\hat{h}(P)=0$.

Lemma 3.8. Suppose $\rho\left(P_{1}, P_{2}, P_{3}\right) \leq 1$. Then $S=\emptyset$.

Proof. First we show that there exists $Q \in E(K) \backslash\{0\}$ with $\hat{h}(Q)=0$ and $f_{v}(Q) \in E_{v}\left(K_{v}\right)_{0}$ for all $v \in S$. If $\hat{h}\left(P_{1}\right)=0$ then we take $Q=P_{1}$ and our claim follows because $S_{1}=\emptyset$. So say $\hat{h}\left(P_{1}\right) \neq 0$. By Lemma 3.7 the global heights coincide $\hat{h}\left(P_{1}\right)=\hat{h}\left(P_{2}\right)$. Since $\rho\left(P_{1}, P_{2}\right) \leq 1$ there are $M, N \in \mathbf{Z}$ not both zero with $[M]\left(P_{1}\right)=[N]\left(P_{2}\right)$. The Néron-Tate height is quadratic, hence $M^{2} \hat{h}\left(P_{1}\right)=$ $N^{2} \hat{h}\left(P_{2}\right)=N^{2} \hat{h}\left(P_{1}\right)$ and thus $M^{2}=N^{2} \neq 0$. So $[M]\left(P_{1} \pm P_{2}\right)=0$ and therefore $\hat{h}(Q)=0$ with $Q=P_{1} \pm P_{2}$. Clearly, $Q \neq 0$ and $f_{v}(Q) \in E_{v}\left(K_{v}\right)_{0} \backslash\{0\}$ for all $v \in S$ because $S_{1}=S_{2}=\emptyset$. 
Now that we have found $Q$ we can easily conclude the proof. Indeed, the Néron local heights of $Q$ can be evaluated by Theorem VI 4.1. Just as in the proof of Lemma 3.7, we use our model with good reduction $E_{v}$ if $v \notin S$ and the Tate curve $E_{q_{v}}$ otherwise. The Néron local heights are non-negative so they all vanish. But a Néron local height coming from a place of bad reduction contributes by a positive term through the vanishing order of the local discriminant. Therefore, $S=\emptyset$.

Lemma 3.9. We have $\rho\left(P_{1}, P_{2}, P_{3}\right) \geq 2$.

Proof. We assume $\rho\left(P_{1}, P_{2}, P_{3}\right) \leq 1$ and deduce a contradiction.

For a certain reordering $(i, j, k)$ of $(1,2,3)$ and fixed $M, N, N^{\prime} \in \mathbf{Z}$ with $M \neq 0$ we have

$$
[N]\left(P_{i}\right)=[M]\left(P_{j}\right) \text { and }\left[N^{\prime}\right]\left(P_{i}\right)=[M]\left(P_{k}\right) .
$$

By the previous lemma we have $S=\emptyset$. So the $j$-invariant of $E$ is a constant $2^{8} 3^{3} a^{3} /\left(4 a^{3}+27 b^{2}\right) \in \mathbf{C}$.

We may reformulate our situation as follows. There exists an irreducible algebraic curve $C$ in the 123-surface for which $\left.j\right|_{C}$ is constant and where relations as in (3.8) hold.

We will prove below that there are infinitely many points on $C$ where the $i$-th coordinate is torsion. The relations (3.8) and Lemma 3.3 lead to a contradiction.

As in the argument stated after Lemma 3.1 there is an elliptic curve $E^{\prime}$ given by (3.1) with $a^{\prime}, b^{\prime} \in \mathbf{C}$ and an isomorphism $E \rightarrow E^{\prime}$ determined by some $w \neq 0$ in an algebraic closure of $K$ satisfying (3.2). We remark $w \notin \mathbf{C}$ because $\mathbf{C}(a, b)$ is not algebraic over $\mathbf{C}$. We may regard $w$ as a non-constant algebraic function on $C$. The image of $P_{i}$ under this isomorphism is $\left(w^{2} i-1 / 12, *\right)$. We may regard it as an algebraic curve in $E^{\prime}$. Now $w^{2} i-1 / 12$ attains, up-to finitely many exceptions, any complex value. In particular, it attains the first coordinate of a torsion point of $E^{\prime}$ infinitely often. This gives the infinitely many points on $C$ with the desired property.

\subsection{There are no torsion anomalous subvarieties}

We now prove Proposition 3.2. First we show that $X$ does not contain any torsion anomalous subvarieties as in part (i) of the definition. Let $C \subset X$ be an irreducible algebraic curve. The coordinate functions $a, b: S \rightarrow \mathbf{A}^{1}$ induce rational functions on $C$. They determine an elliptic curve $E$ defined over $\mathbf{C}(a, b)$ given in Weierstrass $y^{2}=x^{3}+a x+b$. We consider three points $P_{1,2,3}$ as in the previous section. Then $\rho\left(P_{1}, P_{2}, P_{3}\right) \geq 2$ by Lemma 3.9. This means that two independent relations cannot simultaneously hold on $C$. In other words, $C$ cannot be torsion anomalous.

Now we show that $X$ cannot contain a torsion anomalous surface as in part (ii) of the definition. Assuming the contrary, $X$ is a torsion anomalous subvariety of itself. So there is $(\alpha, \beta, \gamma) \in \mathbf{Z}^{3} \backslash\{0\}$ with

$$
[\alpha](1, \sqrt{1+a+b})+[\beta](2, \sqrt{8+2 a+b})+[\gamma](3, \sqrt{27+3 a+b})=0
$$


for all $(a, b) \in S$. We suppose first $\beta \neq 0$ or $\gamma \neq 0$. There is an irreducible algebraic curve $C \subset X$ on which $1+a+b=0$ holds identically. So the first coordinate in $\mathcal{E}$ of a point in $C$ has order 2 . In addition to $(\alpha, \beta, \gamma)$, a second and independent relation $(2,0,0)$ holds on $C$. Therefore, $C$ is torsion anomalous as in part (i) of the definition. This contradicts the already proven part of the proposition. If $\beta=\gamma=0$ we also conclude a contradiction by a similar argument using a curve on which $8+2 a+b=0$ holds.

Finally, by Lemma 3.3 the surface $X$ cannot contain any torsion anomalous subvarieties as in part (iii) of the definition.

\section{Proof of the main result}

Recall that $\mathcal{E}_{L}$ is the Legendre family of elliptic curves over $Y(2)=\mathbf{P}^{1} \backslash\{0,1, \infty\}$ and that $\mathcal{E}$ is the Weierstrass family of elliptic curves over $S=\left\{(a, b) ; 4 a^{3}+\right.$ $\left.27 b^{2} \neq 0\right\}$.

Let $X_{L}$ be an irreducible closed algebraic surface in $\mathcal{E}_{L}^{3}$. In Section 2 we introduced the notion of a torsion anomalous subvariety of $X_{L}$. We call an irreducible closed subvariety of $X_{L}$ a strongly torsion anomalous subvariety of $X_{L}$ if it satisfies (i) or (ii) in the definition of a torsion anomalous subvariety. We write $X_{L}^{\text {sta }}$ for $X_{L} \backslash \bigcup_{A} A$, here $A$ runs over all strongly torsion anomalous subvarieties of $X_{L}$.

Let $X \subset \mathcal{E}^{3}$ be the 123 -surface. We recall that is irreducible. We start off by using it to construct an algebraic surface $X_{L}$ in the Legendre family $\mathcal{E}_{L}^{3}$. We first introduce a covering $S^{\prime}$ of $S$ by setting

$$
\begin{aligned}
S^{\prime}=\left\{\left(a, b, e_{1}, e_{2}, e_{3}, t, r\right) \in S \times \mathbf{A}^{5} ;\right. & e_{i}^{3}+a e_{i}+b=0 \text { for } 1 \leq i \leq 3, \\
& \left(e_{2}-e_{1}\right)^{2}\left(e_{3}-e_{2}\right)^{2}\left(e_{1}-e_{3}\right)^{2} t=1, \\
& \left.e_{2}-e_{1}=r^{2}\right\}
\end{aligned}
$$

Note that $\left(e_{2}-e_{1}\right)^{2}\left(e_{3}-e_{2}\right)^{2}\left(e_{1}-e_{3}\right)^{2}=-\left(4 a^{3}+27 b^{2}\right)$ is invertible in the coordinate ring of $S$. So $t$ as well as $e_{1,2,3}$ and $r$ are integral over the coordinate ring of $S$. In geometric terms this means that the natural projection morphism $S^{\prime} \rightarrow S$ is finite. It is also surjective. The irreducible components of $S^{\prime}$ have dimension 2. We obtain a new Abelian scheme $\mathcal{E}^{\prime 3} \rightarrow S^{\prime}$ by taking the fibered product of $\mathcal{E}^{3} \rightarrow S$ with $S^{\prime} \rightarrow S$. Let $f: \mathcal{E}^{\prime 3} \rightarrow \mathcal{E}^{3}$ be the induced morphism. It is finite and surjective since these properties are preserved under base change. Since $f$ is a closed surjective morphism and $X$ is irreducible, the pre-image $f^{-1}(X)$ contains an irreducible component $X^{\prime}$ with $f\left(X^{\prime}\right)=X$. We must have $\operatorname{dim} X^{\prime}=2$ by standard results in dimension theory, $c f$. Exercise II 3.22 [7].

We define a morphism $S^{\prime} \rightarrow Y(2)$ by $\left(a, b, e_{1}, e_{2}, e_{3}, t, r\right) \mapsto \frac{e_{3}-e_{1}}{e_{2}-e_{1}}$. Then

$$
\left(x, y, a, b, e_{1}, e_{2}, e_{3}, r\right) \mapsto\left(\frac{x-e_{1}}{e_{2}-e_{1}}, \frac{y}{r^{3}}, \frac{e_{3}-e_{1}}{e_{2}-e_{1}}\right)
$$


induces a morphism $g: \mathcal{E}^{\prime 3} \rightarrow \mathcal{E}_{L}^{3}$. Restricted to a fiber of $\mathcal{E}^{\prime 3} \rightarrow S^{\prime}$, it gives an isomorphism between Weierstass and Legendre models of an elliptic curve. Moreover, it fits into the commutative diagram

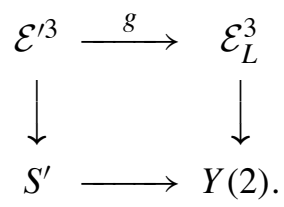

A straight-forward verification shows that $\left.g\right|_{X^{\prime}}: X^{\prime} \rightarrow \mathcal{E}_{L}^{3}$ has finite fibers. The image $g\left(X^{\prime}\right)$ is constructable in $\mathcal{E}_{L}^{3}$ by Chevalley's Theorem. Let $X_{L}$ be the Zariski closure of $g\left(X^{\prime}\right)$ in $\mathcal{E}_{L}^{3}$. Then $X_{L}$ is irreducible and from dimension theory we conclude $\operatorname{dim} X_{L}=2$.

Next, let us show that $X_{L}$ does not contain any torsion anomalous subvariety as in part (ii) of the definition. Indeed, otherwise a non-trivial integral relation would hold identically on $X_{L}$. Any such integral relation would hold on $X^{\prime}$ and also on $X$ because $g$ is fiberwise the cube of an isomorphism of elliptic curves. But no non-trivial integral relation holds on $X$ by Proposition 3.2.

Next, we claim that $X_{L}$ contains only finitely many torsion anomalous subvarieties as in part (i) of the definition. We also claim that each such subvariety intersects $g\left(X^{\prime}\right)$ in only finitely many points.

Let $C \subset X_{L}$ be such a torsion anomalous subvariety. Then $C$ is an algebraic curve and there are two possibilities.

Say first that $\left.g\right|_{X^{\prime}} ^{-1}(C)$ has positive dimension. Then it contains an irreducible algebraic curve $C^{\prime}$. Two independent integral relations hold on $C$. These must continue to hold on $C^{\prime}$. Finally, these relations also hold on $f(C) \subset X$. Latter must have dimension 1 because $f$ is a finite morphism. We have found a torsion anomalous subvariety in $X$ and so a contradiction to Proposition 3.2.

Now say $\left.g\right|_{X^{\prime}} ^{-1}(C)$ has dimension 0 . This implies that $C \cap g\left(X^{\prime}\right)$ is finite. Because $C$ is irreducible it follows that $C$ is in the Zariski closure of $X_{L} \backslash g\left(X^{\prime}\right)$ in $X_{L}$. This closure is a finite union of points and irreducible algebraic curves. Therefore, it contains $C$ as an irreducible component. This leaves only finitely many possibilities for $C$ and our claim above holds.

We have proved that $g\left(X^{\prime}\right) \cap\left(X_{L} \backslash X_{L}^{\text {sta }}\right)$ is finite.

Say $P_{1}, P_{2}, \ldots$ is a sequence of distinct torsion points on $X$. We will deduce a contradiction. Since $\left.f\right|_{X^{\prime}}: X^{\prime} \rightarrow X$ is surjective we find a pre-image, which must be torsion, of each $P_{i}$ in $X^{\prime}$. Because $\left.g\right|_{X^{\prime}}$ has finite fibers, $g\left(X^{\prime}\right)$ contains infinitely many torsion points $Q_{1}, Q_{2}, \ldots$

By the discussion above, only finitely many of the $Q_{1}, Q_{2}, \ldots$ can lie on $X_{L} \backslash$ $X_{L}^{\text {sta }}$. We remove these from our sequence and suppose $Q_{i} \in X_{L}^{\text {sta }}$. By Proposition 2.1(i), only finitely many of the remaining $Q_{i}$ can lie on $X_{L}^{\text {ta }}$. We remove these as well. So $Q_{i} \in X_{L}^{\mathrm{sta}} \backslash X_{L}^{\mathrm{ta}}$.

All $Q_{i}$ are on a torsion anomalous subvariety of $X_{L}$ as in part (iii) of the definition of torsion anomalous. In particular, each $Q_{i}$ is in some fiber with complex 
multiplication. We use Proposition 2.1(ii). After passing to an infinite subsequence, the $Q_{i}$ are all in the same fiber of $\mathcal{E}_{L}^{3} \rightarrow Y(2)$. Let $J \in \mathbf{C}$ be the $j$-invariant of a factor of this fiber. Each of the corresponding $P_{i}$ lies in the cube of an elliptic curve with $j$-invariant $J$. By passing to a infinite subsequence a last time we find infinitely many torsion points on an irreducible algebraic curve in $X$ on which the $j$-invariant is constant. This contradicts Lemma 3.3 and completes the proof of Theorem 1.1.

\section{References}

[1] J. Ax, Some topics in differential algebraic geometry I: Analytic subgroups of algebraic groups, Amer. J. Math. 94 (1972), 1195-1204.

[2] D. Bertrand, Extensions de D-modules et groupes de Galois différentiels, In: " $p$-adic Analysis" (Trento, 1989), Lecture Notes in Math., Vol. 1454, Springer, Berlin, 1990, 125141.

[3] D. Bertrand, Special points and Poincaré bi-extensions, with an Appendix by Bas Edixhoven, arXiv:1104.5178v1 (2011).

[4] E. Bombieri and W. Gubler, "Heights in Diophantine Geometry", Cambridge University Press, 2006.

[5] S. DAVID, Points de petite hauteur sur les courbes elliptiques, J. Number Theory 64 (1997), 104-129.

[6] P. HABegGeR, Special points on fibered powers of elliptic surfaces, J. Reine Angew. Math., to appear.

[7] R. HARTShorne, "Algebraic Geometry”, Springer, 1997.

[8] D. HusemöLleR, "Elliptic Curves", Springer, 2004.

[9] D. W. MASSER and U. ZANNIER, Torsion points on families of squares of elliptic curves, Math. Ann. 352 (2012), 453-484.

[10] D. W. MASSER and U. ZANNIER, Torsion anomalous points and families of elliptic curves, C. R. Acad. Sci. Paris Sér. I 346 (2008), 491-494.

[11] D. W. MASSER and U. ZANNIER, Torsion anomalous points and families of elliptic curves, Amer. J. Math. 132 (2010), 1677-1691.

[12] J. PILA and A. J. Wilkie, The rational points of a definable set, Duke Math. J. 133 (2006), 591-616.

[13] J. PILA and U. ZANNIER, Rational points in periodic analytic sets and the Manin-Mumford conjecture, Atti Accad. Naz. Lincei Cl. Sci. Fis. Mat. Natur. Rend. Lincei (9) Mat. Appl. 19 (2008), 149-162.

[14] R. PINK, A Common generalization of the conjectures of André-Oort, Manin-Mumford, and Mordell-Lang, preprint (2005), 13 pp.

[15] B. Poonen, Spans of Hecke points on modular curves, Math. Res. Lett. 8 (2001), 767-770.

[16] M. Raynaud, Sous-variétés d'une variété abélienne et points de torsion, In: "Arithmetic and geometry”, Vol. I, Progr. Math., Vol. 35, Birkhäuser Boston, Boston, MA, 1983, 327352.

[17] P. RoquetTe, "Analytic Theory of Elliptic Functions over Local Fields”, Hamburger Mathematische Einzelschriften (N.F.), Heft 1, Vandenhoeck \& Ruprecht, Göttingen, 1970.

[18] J. H. Silverman, "Advanced Topics in the Arithmetic of Elliptic Curves", Graduate Texts in Mathematics, Vol. 151, Springer-Verlag, New York, 1994.

[19] J. H. Silverman, "The Arithmetic of Elliptic Curves", Springer, 1986. 
[20] L. VAN DEN DRIES, A generalization of the Tarski-Seidenberg theorem, and some nondefinability results, Bull. Amer. Math. Soc. (N.S.) 15 (1986), 189-193.

[21] L. VAN DEN DRIES, "Tame Topology and o-minimal Structures", London Mathematical Society Lecture Note Series, Vol. 248, Cambridge University Press, Cambridge, 1998.

[22] B. ZILBER, Exponential sums equations and the Schanuel conjecture, J. London Math. Soc. (2) 65 (2002), 27-44.

Technische Universität Darmstadt

Fachbereich Mathematik

Schlossgartenstrasse, 7

64289 Darmstadt, Germany

habegger@mathematik.tu-darmstadt.de 